Chimia 46 (1992) 200-214

(C) Neue Schweizerische Chemische Gesellschaft ISSN 0009-4293

\section{In Situ Infrared Attenuated Total Reflection (IR ATR) Spectroscopy: A Complementary Analytical Tool for Drug Design and Drug Delivery}

\author{
Urs Peter Fringeli*
}

Abstract. A comprehensive summary of basic relations for quantitative IR ATR spectroscopy of isotropic and oriented samples is given. Experimental requirements for detection of sub-monolayer quantities in aqueous environment are discussed. New instrumental developments such as a single-beam-sample-reference (SBSR) attachment, and FTIR modulation spectroscopy at low frequencies are presented. Examples of in situ experiments with tertiary-amine local anesthetics interacting with planar, immobilized lipid bilayers are discussed. Partition coefficients of the total amine, as well as of the protonated and deprotonated forms have been determined. Structural alterations, especially of lipids, were detected upon interaction. Adsorption isotherms revealed multilayer formation at the membrane surface. The onset of this process for dibucaine is at ca. $5 \mathrm{~mm}$ bulk concentration or even below. Preferential accumulation of dibucaine base is observed already at bulk $\mathrm{pH} 5.5\left(\mathrm{p} K_{\mathrm{a}}=8.83\right)$. For peptide conformation analysis, FTIR temperature $(T)$ modulation experiments were performed for the first time. A modulation amplitude of $\Delta T= \pm 1^{\circ}$ at a given mean temperature is shown to result in high-quality phase-resolved spectra enabling detailed insight into periodically induced changes of the state of the sample.

\section{Introduction}

In the past decade, Fourier-transform IR (FTIR) spectrometers have replaced dispersive instruments because of their better performance in nearly all respects [1]. The problem of background compensation e.g. could be reduced significantly, enabling routine measurements even in aqueous environment. Therefore, a growing interest in IR membrane spectroscopy was observed from the biophysical, biochemical, and biomedical viewpoints [239] as well as from the biosensor part [40][41]. The works cited here have to be considered as typical examples just available to the author at present. Since the aim of this article is presenting ATR tech-

\footnotetext{
*Correspondence: Prof. U.P. Fringeli University of Vienna Währingerstrasse 42 A-1090 Vienna and Institute for Physical Chemistry ETH-Zentrum CH-8092 Zürich
}

niques for in situ experiments as well as the theoretical background for quantitative analysis and orientation measurement, rather than reviewing papers published so far, the reader is also referred to the reference lists of cited papers. In most of these papers conventional transmission $(\mathrm{T})$ technique has been used, perhaps because for simple applications sample preparation and data interpretation may be easier than with ATR technique. The latter, however, has significant advantages over $\mathrm{T}$ spectroscopy, especially with respect to structure determination under in situ conditions.

Sect. 2 gives a comprehensive introduction into the principles of ATR spectroscopy, wheras Sect. 3 deals with experimental considerations, such as sensitivity requirements, background compensation and modulation spectroscopy. Sect. 4 gives a summary of typical applications like preparation of model membranes and living cells for in situ experiments, interaction of local anesthetics with lipid bilayers, determination of partition coefficients between membrane and aqueous environ-

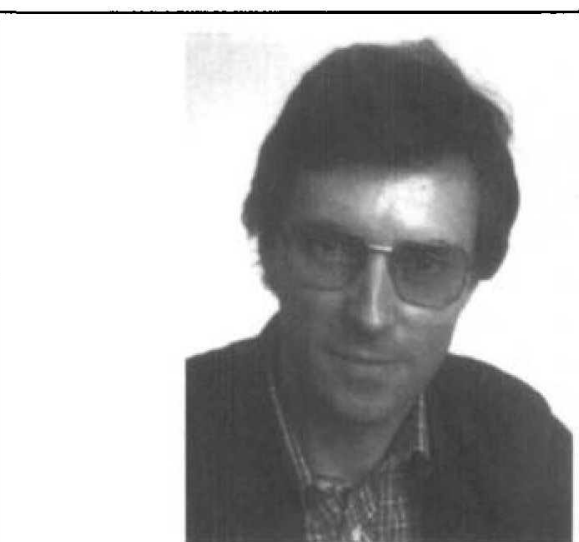

Urs Peter Fringeli. Born November 26, 1939 in Basel, Switzerland. 1959-63 Study of Natural Sciences at the ETH-Zürich. Diploma work in Physical Chemistry. 1964-69: Doctorate at the Laboratory for Physical Chemistry of ETH with Prof. Hs. H. Günthard. Thesis: 'Construction of an Infrared-Modulationsorption and Life-time of Photochemically low at the Max-Planck-Institute for Biophysical Chemistry, Göttingen (Prof. M. Eigen), and at the Battelle Institute, Geneva (Prof. $W$. Leuzinger). 1972-83: Project leader in Prof. Günthard's group at the Laboratory for Physical Chemistry, ETH-Zürich. Field: Molecular Structure of Biomembranes. Method: Infrared-ATR Spectroscopy, Lectures in Biophysical Chemistry. 1977: Privatdozent, 1979: Ruzicka award of the Council of Swiss Federal Institutes of Technology. Since 1984: Professor for Applied Physical Chemistry and Biophysical Chemistry at the University of Vienna. Academic guest at the Laboratory for Physical Chemistry of ETH-Zürich. Spectrometer for the Measurement of $\mathrm{Ab}$ Excited States.' 1970-72: Postdoctoral fel-

ment, as well as temperature modulation. The latter enables access to conformational equilibria of polypeptids and lipids. Besides of utmost background compensation, modulation spectroscopy enables $\mathrm{ki}$ netic analysis, too, provided that the circular modulation frequency $\omega_{\mathrm{m}}$ and the rate constants of excited reactions are of the same magnitude. The situation is optimum, when the response of the sample is fully reversible [42-46]. Estimates reveal that signal to noise $(\mathrm{S} / \mathrm{N})$ enhancement by this technique should be sufficient to get information on the molecular structure of a substrate molecule bound to its specific receptor under in situ conditions. Such data are of predominant interest in drug design.

\section{Principles of ATR Spectroscopy}

\subsection{Basic Phenomena}

Fig. 1 shows a schematic comparison of the conventional transmission $(a)$ and the internal reflection $(b)$ technique. The latter is achieved by placing the sample 
material in close contact with the optically transparent internal reflection element (IRE) of higher refractive index and working above the critical angle $\theta_{c}$.

Under these conditions, it follows that an electromagnetic field still exists in the rare medium beyond the reflecting interface. This field exhibits the frequency of the incoming light, but the amplitude falls off exponentially with distance $z$ from the surface.

$$
E=E_{\mathrm{o}} e^{-z / d \mathrm{p}}
$$

$d_{p}$ denotes the depth of penetration and is given by

$$
d_{\mathrm{p}}=\frac{\lambda_{1}}{2 \pi\left(\sin ^{2} \theta-n_{21}^{2}\right)^{1 / 2}}
$$

$n_{21}$ stands for the ratio $n_{2} / n_{1}$ of the refractive indices of the rarer medium $\left(n_{2}\right)$ and of the internal reflection element $\left(n_{1}\right) . \lambda_{1}$ denotes the wavelength $\lambda_{\text {vakuum }} / n_{1}$ in the latter. According to Eqn. 2, the penetration depth amounts to the order of magnitude of the wavelength $\lambda_{1}$ of the IR radiation in the IRE. Fig. 2 schematically represents the situation when a thin film (e.g. a bilayer or a protein layer) is immobilized at the surface of the IRE. This is a typical set-up for in situ membrane and biosensor studies. The displacement $D$, the so-called Goos-Hänchen displacement [48] is also in the order of $\lambda_{1}$.

The 'evanescent wave' exhibits another interesting feature, namely the existence of electric field components along all three axis of the rectangular coordinate system attached to the ATR plate (Fig. 3). In contrast, the incident plane wave has only electric field components perpendicular to the direction of propagation. By means of a polarizer, two distinct directions of the incident electric-field vector may be chosen, namely parallel ( $\|, \mathrm{pp})$ and perpendicular $(\perp, v p)$ to the plane of incidence ( $x, z$ plane). For geometrical reasons (see Fig. 3) it follows that $E_{\|}$results in the $E_{x}$ and $E_{z}$, and $E_{\perp}$ the $E_{y}$ component of the evanescent field.

The relative electric field components in the rarer medium 2 at $z=0(1,2$ interface), i.e. the ratio between the electricfield components $E_{02 x}, E_{02 z}, E_{02 y}$ and $E_{0111}$, $E_{01 \perp}$ are given by Fresnel's equations [49]. Eqns. 3-5 hold exactly for non-absorbing media 1 and 2, but they are good approximations for weak absorbing samples [46][47]. This condition is fulfilled for all types of experiments reported in this article [46].

The relative electric field components in a thin film of thickness $d<<d_{\mathrm{p}}$ (see Fig. 2) are given by:

$$
\begin{aligned}
& E_{02 x}^{r}=E_{02 x} / E_{0111}=\frac{2 \cos \theta\left(\sin ^{2} \theta-n_{31}^{2}\right)^{1 / 2}}{\left(1-n_{31}^{2}\right)^{1 / 2}\left[\left(1+n_{31}^{2}\right) \sin ^{2} \theta-n_{31}^{2}\right]^{1 / 2}} \\
& E_{022}^{r}=E_{022} / E_{0111}=\frac{2 \cos \theta n_{32}^{2} \sin \theta}{\left(1-n_{31}^{2}\right)^{1 / 2}\left[\left(1+n_{31}^{2}\right) \sin ^{2} \theta-n_{31}^{2}\right]^{1 / 2}} \\
& E_{02 y}^{r}=E_{02 y} / E_{01 \perp}=\frac{2 \cos \theta}{\left(1-n^{2}{ }_{31}\right)^{1 / 2}}
\end{aligned}
$$

$$
\text { with } E_{02 \|}^{r}=\left(E_{02 x}^{r^{2}}+E_{02 z}^{r^{2}}\right)^{1 / 2} \text {, and } E_{02 \perp}^{r}=E_{02 y^{*}}^{r} \text {. }
$$

$n_{i k}$ denotes the ratio of refractive indices $n_{i} / n_{k}$ of medium $i$ and $k$, where $i, k=1,2$, and 3 stand for ATR plate, thin films and surrounding medium, respectively. For bulk rarer medium $d \gg d_{\mathrm{p}}$ (see Fig. 2) index 3 must be replaced by 2 . It should be noted, that generally $E_{0 x}^{r}, E_{0 y}^{r}$, and $E_{0 z}^{r}$ are not equal.

The relative electric-field strength for intermediate layer thickness $\left(d \approx d_{\mathrm{p}}\right)$ may be approximated by the Interpolation 6 .

$$
\begin{aligned}
& E_{02}^{r}(d)=E_{02}^{r}(\mathrm{th})+\left(1-e^{-d / d} \mathrm{p}\right) \\
& \left(E_{02}^{r}(\mathrm{bu})-E_{02}^{r}(\mathrm{th})\right)
\end{aligned}
$$

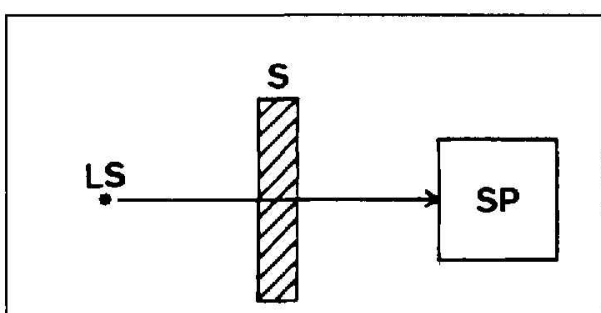

a. TRANSMISSION

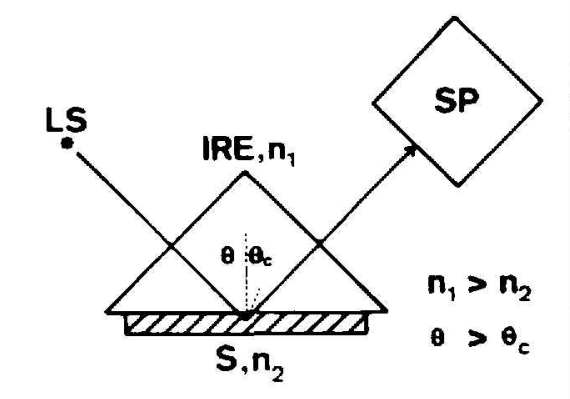

\section{b. INTERNAL REFLECTION}

Fig. 1. Comparison of transmission (a) with inter nal reflection (b) technique. LS: light source, $\mathrm{S}$ : sample, SP: spectrometer, IRE: internal reflection element (ATR crystal), $\theta$. angle of incidence, $\theta_{c}$ : critical angle, $n_{1}, n_{2}:$ refractive index of IRE and $S$, respectively. where th and bu denote 'thin layer', and 'bulk', respectively. For $d \rightarrow 0$ Eqn. 6 results in Eqns. 3-5 for thin layers, and for $d \rightarrow \infty$ one obtains the electric-field components in a bulk rarer medium (Eqns. 35 with $n_{3}=n_{2}$ ).

\subsection{Quantitative Analysis of Isotropic Samples}

Quantitative analysis in transmission spectroscopy is based on Lambert-Beer's law

$$
T=I_{\mathrm{S}} / I_{0}=10^{-\mathrm{A}}=10^{-\operatorname{ecd}}
$$

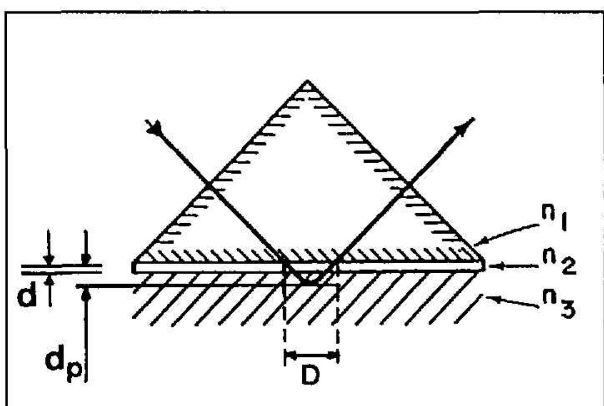

Fig. 2. Measurements on thin films. The penetration depth $d_{\mathrm{p}}$ is larger than the film thickness $d_{.} n_{1}$, $n_{2}, n_{3}$ denote the refractive indices of IRE, film and bulk environment, respectively. The penetrating ray is displaced by the distance $D$ [48].

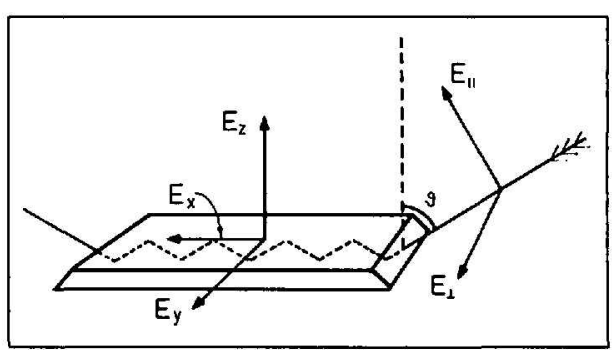

Fig. 3. ATR set-up for multiple internal reflections $(M I R) . \theta$, angle of incidence; $E_{\|,} E_{\perp}$, parallel and perpendicular polarized components of the electric field of incident light. $E_{x}, E_{y}, E_{z}$, electricfield components with respect to the plate-fixed coordinate system. $\left(E_{\|} \rightarrow E_{x}, E_{z}, E_{\perp} \rightarrow E_{y}\right.$, see [46][47]). From [2]. 
The meaning of the symbols are transmittance $(T)$, intensity before $\left(I_{0}\right)$ and after $\left(I_{S}\right)$ the sample, absorbance $(A)$, molar absorption coefficient $(\varepsilon)$, molar concentration $(c)$, and sample thickness $(d)$, respectively. To apply Lambert-Beers's law to ATR data, one has to introduce a hypothetical thickness $d_{\mathrm{e}}$ of a sample which would result in the same absorbance of a given band via transmission as obtained with the real sample thickness $d$ in the ATR experiment. The quantity $d_{\mathrm{e}}$ was introduced by Harrick [47] and referred to as 'effective thickness'.

For the limiting cases 'bulk medium 2' $(d=\infty)$, and 'thin layer' $(d<<d)$ one nhtains

$$
\begin{aligned}
& d_{e}=\frac{1}{\cos \theta} \frac{n_{2}}{n_{1}} \frac{d_{\mathrm{p}}}{2} E_{02}^{r^{2}} \text { and } \\
& d_{\mathrm{e}}=\frac{1}{\cos \theta} \frac{n_{2}}{n_{1}} d E_{02}^{r^{2}}, \text { respectively (9) }
\end{aligned}
$$

It should be noted that $d_{\mathrm{e}}$ for a bulk medium increases linearly with wavelength $\lambda$ (see Eqn. 2), whereas $d$ for a thin film does not depend on $\lambda$. The effective thickness for an intermediate sample thickness $\left(d \approx d_{\mathrm{p}}\right)$ may be calculated by using Eqn.6. For more details, on effective thickness, see [46]. As a consequence of the application of Fresnel's equations, simple application of Lambert-Beer's law to ATR data requires polarized incident light [46],

$$
\begin{aligned}
& T_{\|}=10^{-e c d_{\mathrm{ell}}}=10^{-A_{\|}}, \text {and } \\
& T_{\perp}=10^{-e c d_{\mathrm{e} \perp}=10^{-A_{\perp}}}
\end{aligned}
$$

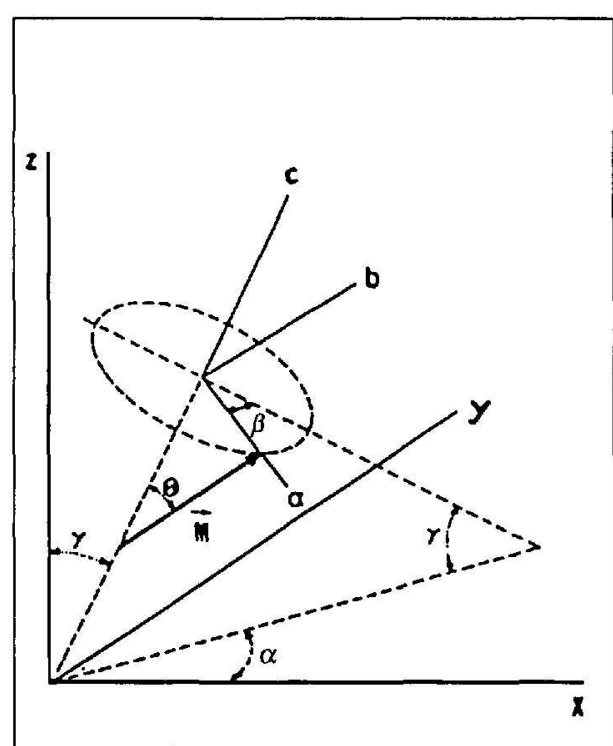

Fig. 4. Partial axial orientation. Molecular axis forms angle $\gamma$ with $z$ axis of the ATR plate-fixed coordinate system $x, y, z . a, b, c$ is the moleculefixed coordinate system. $\alpha, \beta$, and $\gamma$ are the Eulerian angles. From [51].

\subsection{Quantitative Analysis of Oriented Samples}

In the case of an oriented sample, the absorbance depends not only on the number of particles per volume (or area) but also on the structure of the sample. For that reason, quantitative analysis of an oriented sample means both, determination of concentration and orientation.

\subsubsection{Orientation Measurements}

For a comprehensive discussion of orientation measurements in transmission spectroscopy, see [50-52].

\subsubsection{Theory of Integrated Absorbance of Oriented Samples}

The integrated absorbance is proportional to the square of the scalar product between the transition dipole moment $\vec{M}$ and the electric field $\vec{E}$ of radiation. For the case of excitation of one quantum of the $\mathrm{k}$-th normal mode $q_{\mathrm{k}}$ from the ground state, $\vec{M}$ is proportional to the change ( $\delta \vec{\mu}$ $\left./ \delta q_{k}\right)_{0}$ of the molecular dipole moment $\vec{\mu}$ with respect to the normal mode displacement $q_{2}$ [53].

\section{$A(\tilde{v}) \mathrm{d} \tilde{v}=c d \cdot \int \varepsilon(\tilde{v}) \mathrm{d} \tilde{v} \alpha(\vec{M} \cdot \vec{E})^{2},(11)$}

band

where

$$
(\vec{M} \cdot \vec{E})^{2}=|\vec{M}|^{2}|\vec{E}|^{2} \cos ^{2}(\vec{M}, \vec{E})=
$$$$
\left(M_{x} E_{x}+M_{y} E_{y}+M_{z} E_{z}\right)^{2}
$$

Eqns. 11 and 12 form the basis of orientation measurements.

\subsubsection{Ultrastructure}

Two problems have to be solved in order to get structural information. First, the relative orientation of the ATR plate fixed coordinate system $(x, y, z)$ and the molecular coordinate system $(a, b, c)$ must be known. This is equivalent to the knowledge of the ultrastructure of the molecular entity. Secondly, the knowledge of the orientation of the transition dipole moment $\vec{M}\left(M_{a}, M_{b}, M_{c}\right)$ within the molecule is a prerequisite for molecular-structure analysis. This requirement often limits the application of the analytical procedure described below to typical vibrations of functional groups, unless more details on the direction of $\vec{M}$ are available via normal coordinates analysis [53]

The angles $\alpha, \beta$ and $\gamma$ are the Eulerian angles, enabling description of the mutual position of the two coordinate systems. Transformation of $\left(M_{a}, M_{b}, M_{c}\right)$ into $\left(M_{x}, M_{y}, M_{z}\right)$ is performed by the matrix Eqn. 13 .

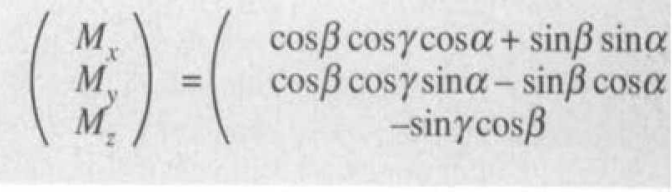

Thus, if $\left(M_{a}, M_{b}, M_{c}\right)$ are known from experience or from normal coordinates analysis [53] $\left(M_{x}, M_{y}, M_{z}\right)$ are calculated by coordinate transformation (13) and inserted into Eqns. 11 and 12 . The electric-field components are given by Eqns. 3-6. Obviously, this procedure results in a quantity which is proportional to the integrated absorbance, however, for most samples this is only a fractional contribution, since the entity of molecules exhibit a certain distribution with respect to the Eulerian angles, which is typical for a given ultrastructure. For definition of ultrastructures see [45][46]. Typical features of biomembranes are random arrangement of molecules around a space-fixed axis normal to the plane of the membrane ( $z$ axis) and free rotation around the molecular axis ( $c$ axis) as well as fluctuation of the angle $\gamma$ between molecular axis and spacefixed axis (Fig. 4). This ultrastructure is referred to as liquid crystalline ultrastructure (LCU) and is characterized by isotropy with respect to $\alpha$ and $\beta$. whereas $\gamma$ exhibits a distribution around a mean value $\gamma_{0}\left(0^{\circ} \leq \gamma \leq 90^{\circ}\right)$.

The fluctuation of the molecular axis (c) with respect to the space fixed axis $(z)$ is described by the distribution function $f(\gamma)[51][52][54]$

\subsubsection{Dichroic Ratio and Order Parameter}

The relevant parameter in most optical orientation measurements is the dichroic ratio $\mathrm{R}$

$$
R=\frac{A_{\|}}{A_{\perp}}=\frac{\int A_{\|}(\bar{v}) \mathrm{d} \bar{v}}{\int A_{\perp}(\bar{v}) \mathrm{d} \bar{v}}
$$

$A_{\|}$and $A_{\perp}$ denote the peak absorbances of a given band for parallel (II) and perpendicular $(\perp)$ polarized incident light, respectively. Following the path described above, the axial absorbances $A_{x}, A_{y}$, and $A_{z}$ can be calculated for a given ultrastructure. The procedure has been described in details for transmission spectroscopy by Zbinden [51] and Michl and Thulstrup [52]. Adaptation to ATR spectroscopy is straightforward and has been done selecting the $y$ axis [55] and the $z$ axis [45][46] as space-fixed axis of a LCU. In the latter case, the axial absorbances are given by 


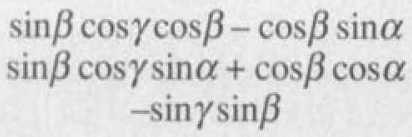

samples aligned along the $z$ axis. The relevant quantity for orientation measurement was reported to be $f=1 / 2\left(3 \cos ^{2} \beta\right.$ $1)$ and referred to as 'order parameter'. $\beta$ denotes the mean angle between the $z$ axis and the molecular axis. From the definition of the order parameter (Eqn. 18), which is the same as in [38], it follows that the expression $f=1 / 2\left(3 \cos ^{2} \beta-1\right)$ can only result for a distribution function $\delta(\gamma-\beta)$ (see Eqn. 18). This means, however, that any molecular fluctuation is excluded, which is equivalent to setting the order parameter $S_{z z}=S=1$ (Eqn. 19). Analysis of polarized ATR spectra of LCU systems by the formalism given in [38] may, therefore, lead to significant systematic errors. Let e.g. $R_{1}$ and $R_{2}$ be the dichroic ratios of equal functional groups at different positions in a molecule. $R_{1}=R_{2}$ only means same orientation for both groups, if $S_{1}=$ $S_{2}$. If, however, $S_{1} \neq S_{2}$, which means
$\Theta$ and $N$ denote the angle between the tansition moment $\vec{M}$ and the molecular axis $c$, and the number of active internal reflections, respectively. The parameter $S$, the so called order parameter, is defined according to (18)

$$
S=\frac{3}{2} \int_{0}^{\pi / 2} f(\gamma) \cos ^{2} \gamma \mathrm{d} \gamma-\frac{1}{2}
$$

$f(\gamma)$ is the distribution function (probability density) for the molecular axis $c$ (see Fig. 4) with respect to a space fixed axis (e.g. $z$ axis). Since an isotropic sample exhibits $f(\gamma)=\sin \gamma$, it follows from Eqn. 18 that the corresponding order parameter becomes zero $\left(S_{\text {iso }}=0\right)$. On the other hand, a perfect alignment of the molecular axis along the $z$ axis results in $f(\gamma)=\delta(\gamma)$, leading to a corresponding order parameter of $\mathbf{S}=1$. Fluctuation of the $c$ axis with respect to the $z$ axis will reduce the order parameter correspondingly. Thus, for a LCU it follows $0<S<1$. It should be noted that, in a more general picture [56-58], $S$ corresponds to the diagonal element $S_{z z}$ of the order parameter tensor $S$. For LCU one obtains

$S=S_{z z}=-2 S_{x x}=-2 S_{y y}$

which is confirmed by Eqns. 15-17. Introducing Eqns. $15-17$ into Eqn. 14 by taking into account that $A_{\|}=A_{x}+A_{z}$, and $A_{\perp}=A_{y}$, one obtains for the dichroic ratio with respect to the $z$ axis

$$
R_{z}^{A T R}(\mathrm{LCU})=\frac{E_{02 x}^{r^{2}}}{E_{02 y}^{r^{2}}}+\frac{E_{02 z}^{r^{2}}}{E_{02 y}^{r^{2}}} \frac{1+S\left(3 \cos ^{2} \Theta-1\right)}{1-\frac{1}{2} S\left(3 \cos ^{2} \Theta-1\right)}
$$

Finally, it should be mentioned that structural features as revealed by Eqns. $15--17$ and Eqn. 20 are expressed by the orientation function

$\sigma=S\left(3 \cos ^{2} \Theta-1\right)$

As a consequence, in a LCU picture as descirbed above determination of both, $S$ and $\Theta$ is not possible by a single dichroic ratio of a given band. It requires the analysis of the dichroic ratios of at least two absorption bands $(1,2)$ resulting from the same functional group. Since in this case $S_{1}=S_{2}=\mathrm{S}$, and in a given (assumed) orientation $\Theta_{1}$ and $\Theta_{2}$ are related for geometrical reasons, it is possible to determine both, the order parameter (fluctuation) and the mean spacial orientation of the corresponding functional group. Good estimates of the direction of $\vec{M}\left(M_{a}, M_{b}\right.$, $M_{c}$ ) (see Fig. 4) are known for a number of functional groups [45], such as $v_{s}\left(\mathrm{CH}_{2}\right)$, $v_{o s}\left(\mathrm{CH}_{2}\right), \delta\left(\mathrm{CH}_{2}\right), \gamma_{w}\left(\mathrm{CH}_{2}\right), \gamma_{r}\left(\mathrm{CH}_{2}\right)$; $v_{a s}\left(\mathrm{COO}^{-}\right), v_{s}\left(\mathrm{COO}^{-}\right) ; v(\mathrm{C}=\mathrm{O}), v(\mathrm{COO})$; $v_{a s}\left(\mathrm{PO}_{2}^{-}\right), v_{s}\left(\mathrm{PO}_{2}^{-}\right)$etc. Combination of Eqns. 20 and 21 results in

$$
R_{z}^{A T R}(\mathrm{LCU})=\frac{E_{02 x}^{r^{2}}}{E_{02 y}^{r^{2}}}+\frac{E_{02 z}^{r^{2}}}{E_{02 y}^{r^{2}}} \frac{1+\sigma}{1-\frac{1}{2} \sigma}
$$

In a recent paper, Hübner and Mantsch [38] have derived a formalism for the analysis of polarized ATR spectra of LCU different fluctuation behaviour, one has to conclude different spacial orientations. On the other hand, if $R_{1} \neq R_{2}$ and $S_{1} \neq S_{2}$ both groups may still have the same mean orientation. Consequently, the orientational accuracy claimed in [38] is not substanciated.

\subsubsection{Dichroic Ratio of Overlapped Bands} i) Different Degrees of Ordering

A situation often found in polypeptideand hydrocarbon-chain spectroscopy is a superposition of typical absorption bands (e.g. amide I,II, $\mathrm{CH}_{2}$ stretching, bending, rocking). This leads to an overall dichroic ratio which is experimentally accessible. It depends on the orientation of each associated functional group. Separation by any line-shape analysis procedure is mostly not possible. A good estimation of the amount and orientation of one component can be obtained, however, when mole fraction and orientation of the other components are known [59-61]. Eqn. 23 is at least a good tool to calculate the dichroic ratio of a composed band i.e. to assess an

expected molecular conformation with respect to its consistency with experimental data. 


$$
R_{z}^{A T R}=\frac{A_{\|}}{A_{\perp}}=\frac{\sum_{i=1}^{n} x_{i} m_{i}^{2}\left\{E_{02 x}^{r^{2}}\left[1-\frac{1}{2} \sigma_{i}\right]+E_{02 z}^{r^{2}}\left[1+\sigma_{i}\right]\right\}}{\sum_{i=1}^{n} x_{i} m_{i}^{2} E_{02 y}^{r^{2}}\left[1-\frac{1}{2} \sigma_{i}\right]}
$$

$x_{i}$ and $m_{i}$ denote the mole fraction and the relative transition moment $\left(m_{i}=\left|\vec{M}_{i}\right| /\left|\vec{M}_{1}\right|\right)$.

$\sigma_{i}$ is the orientation function of the $i$-th $W_{z}=1+S\left(3 \cos ^{2} \Theta-1\right)=1+\sigma$ segment as defined by Eqn. 21 .

\section{ii) Dichroic Difference Spectra}

This is a method which enables subtraction of the random component from the whole band. The procedure is of general practical interest, because it helps localizing orientated regions in a molecule. Dichroic difference spectra $A^{*}(\tilde{v})$ are weighted difference spectra between parallel polarized $A_{\|}(\tilde{v})$ and perpendicular polarized $A_{\perp}(\tilde{v})$ absorbance spectra of the same sample, i.e.

$$
A^{*}(\tilde{v})=A_{\|}(\tilde{v})-R_{\text {iso }} A_{\perp}(\tilde{v})
$$

where $R_{\text {iso }}$ denotes the dichroic ratio of an isotropic sample. Obviously, in the dichroic difference spectrum $A^{*}(\tilde{v})$, all absorption bands resulting from isotropically oriented transition dipole moments are eliminated. Eqn. 24 has been used for the subtraction of the random part of protein spectra [2][28][60]. Details on the practical procedure are given in [46]

\subsection{Determination of Surface Concen- tration}

The concept of 'effective thickness' (see above) is maintained also for oriented samples. However, one has to take into account that the effective thickness may become zero (no absorbance), if the angle between the transition dipole moment $\vec{M}$ and the electric-field vector $\vec{E}$ is $90^{\circ}$, and maximum for parallel alignment of the two vectors. Weighting is achieved by multiplying the axial effective thicknesses obtained for an isotropic sample by factors depending on the ultrastructure of the sample.

In the case of LCU with predominant alignment along the $z$ axis, the axial absorbances $A_{x}, A_{y}$, and $A_{z}$ are given by Eqns. 15-17 with $A_{\|}=A_{x}+A_{z}$ and $\mathrm{A}_{\perp}=A_{y} \cdot A_{\|}$ and $A_{\perp}$ are experimentally available. $\mathrm{Ob}$ viously, the expressions within brackets are the typical features of LCU/z. The weighting factors for axial effective thicknesses of an oriented sample are, therefore, given by

$W_{x y}=1-\frac{1}{2} S\left(3 \cos ^{2} \Theta-1\right)=1-\frac{1}{2} \sigma(25)$

and

Eqns. 25 and 26 become unity in the isotropic case $S=0$. Therefore,

$$
\begin{aligned}
& d_{e x}=W_{x y} d_{e x}^{\text {iso }}, d_{e y}=W_{x y} d_{e y}^{\text {iso }} \\
& d_{e z}=W_{z} d_{\mathrm{ez}}^{\text {iso }}
\end{aligned}
$$

with

$d_{e l l}=d_{e x}+d_{e z}$, and $d_{e \perp}=d_{e y}$

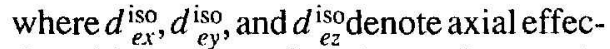
tive thicknesses of an isotropic sample (Eqns. 8 and 9). For the concentration of an oriented sample one obtains

$$
\begin{aligned}
& c=\frac{A_{\|}}{v N \varepsilon d_{\mathrm{ell}}}=\frac{A_{\perp}}{v N \varepsilon d_{\mathrm{e} \perp}} \text {, or } \\
& c=\frac{\int A_{\|} \mathrm{d} \tilde{v}}{v N \int \varepsilon \mathrm{d} \tilde{v} d_{e l l}}=\frac{\int A_{\perp} \mathrm{d} \tilde{v}}{v N \int \varepsilon \mathrm{d} \tilde{v} d_{e \perp}} \text {, or }
\end{aligned}
$$

for peak absorbances, or integrated absorbances (band areas), respectively. $\int \varepsilon d \bar{v}$ is the integral molar absorption coefficient. Use of integrated absorbances is recommended in all cases, where band broadening or narrowing must be expected, e.g. due to conformational changes. $v$ and $N$ denote the number of equal functional groups per molecule, and the number of active internal reflections, respectively.

In the case of thin layers ( $\left.d<d_{\mathrm{p}}, F i g .2\right)$, it is often more adequate to use the surface concentration $\Gamma$ instead of the molarity $c$. $\Gamma$ is obtained from Eqns. $29 a, b$ simply by multiplication by the layer thickness $d$.

$\Gamma=c d$

\section{Experimental Considerations}

\subsection{Sensitivity Requirements for in situ Measurements}

Optimum Number of Internal Reflections

Considering the symmetric $\mathrm{CH}_{2}$ stretching $\left(v_{s}\left(\mathrm{CH}_{2}\right)\right)$ band of a phospholipid monolayer, one should expect an absorptance of about $0.2 \%$ per internal reflection. From this point of view, 100 or even more internal reflections would be desirable. However, these mono and submonolayer absorptions have to be detected in aqueous environment which itself is a strongly absorbing medium (see Fig. 5). The latter requires as few internal reflections as possible. Optimization [46] resulted in $N=20-40$ for a germanium IRE, and 10-20 for a ZnSe IRE (angle of incidence: $\Theta=45^{\circ}$.

\subsection{Significance of Liquid Water Com- pensation}

The vibrational spectrum of liquid water (see Fig. 5 and [46]) is commonly the most intense part of the background to be compensated in IR spectroscopy of biological systems. Unfortunately, the amide-I band of peptides and proteins, which are sensitive to the secondary structure are completely overlapped by the $\mathrm{H}_{2} \mathrm{O}$ bending $\left(\delta\left(\mathrm{H}_{2} \mathrm{O}\right): \sim 1640 \mathrm{~cm}^{-1}\right)$. In many papers published so far, access to amide I was performed by subtraction of the water background by means of the spectrometer software. Structural information was then obtained by application of Fourier self-deconvolution and/or

curve-fitting techniques, to separate the amide-I band into components typical for secondary structural elements, such as $\alpha$ helix, pleated sheet, $\beta$ turns, random. These results, however, are of little confidence, because bulk water and bound water exhibit different peak wavenumbers (up to $20 \mathrm{~cm}^{-1}$ ) and different molar absorption coefficients (up to a factor of 10) [46]. As a consequence, one should be aware that bound water is an integral part of the sample. It cannot be subtracted, at least not by manual manipulations with a bulk water reference spectrum. For a more detailed discussion of this fundamental problem, see [46].

\subsection{ATR Attachment}

Single-Beam-Sample-Reference (SBSR)

\section{Technique}

As a consequence of the required minimum number of internal reflections for monolayer spectroscopy on the one hand, and of the strongly absorbing background on the other hand, the spectral regions of $\mathrm{OH}$ stretching $\left(\sim 3400 \mathrm{~cm}^{-1}\right)$ and OD stretching $\left(\sim 2500 \mathrm{~cm}^{-1}\right)$ are completely blocked, depending on whether $\mathrm{H}_{2} \mathrm{O}$ or $\mathrm{D}_{2} \mathrm{O}$ is used as solvent. The transmittance 
of the corresponding bending vibrations $\left(\delta\left(\mathrm{H}_{2} \mathrm{O}\right): \sim 1640 \mathrm{~cm}^{-1} ; \delta\left(\mathrm{D}_{2} \mathrm{O}\right): \sim 1200\right.$ $\left.\mathrm{cm}^{-1}\right)$ is only a few percent under these conditions. A significant compensation of such strong bands is a prerequisite for in situ spectroscopy, and requires a good reproducibility of the optical path of the IR beam in the multiple internal-reflection element (MIRE), especially for corresponding sample and reference measurements. Moreover, a well-defined angle of incidence and number of internal reflections are important for quantitative analysis. We have achieved best results with dispersive [60] [63-68] and Fourier transform (FT) instruments [46][61][62][105] by a single trapezoid MIRE (Fig. 6) for sample and reference, as well.

Using parallel incident light focused to the entrance face of the crystal by means of a cylindrical mirror, it was possible to place the sample, e.g. at the lower half, and the reference at the upper half of the MIRE. The IR beam is directed alternatively through sample and reference side. In dispersive instruments, a rotating chopper wheel with two rows of different numbers of holes (see Fig. 7) was used in order to label sample and reference beams with different frequencies.

Because sample and reference are measured with a single beam without cell displacement, this method is referred to as Single-Beam-Sample-Reference (SBSR) technique. The most obvious advantage of this attachment is the lack of problems with atmospheric $\mathrm{H}_{2} \mathrm{O}$ and $\mathrm{CO}_{2}$ absorptions. SBSR spectra exhibit also a better long-term stability than generally achieved in the single-beam mode [46]. Furthermore, SBSR technique enables ad hoc changes of the reference environment without problems, since there is no need for separate reference spectra which might require detachment of the ATR cell. The latter would be necessary in the singlebeam (SB) mode, leading to a decrease of the accuracy of background compensation.

\subsection{Modulation Spectroscopy}

\subsubsection{Introduction}

A situation often encountered in chemical and biological IR spectroscopy is the detection of small structural differences on a molecular level, induced in a system by the change of external parameters, as the concentration of an agent, temperature, irradiation, electric field, etc. Conventionally, this information is obtained by measuring the IR spectra at two different parameter settings and then calculating the difference spectrum. The smaller this difference is, however, the more critical is the influence of system instabilities occurring between the two measurements.

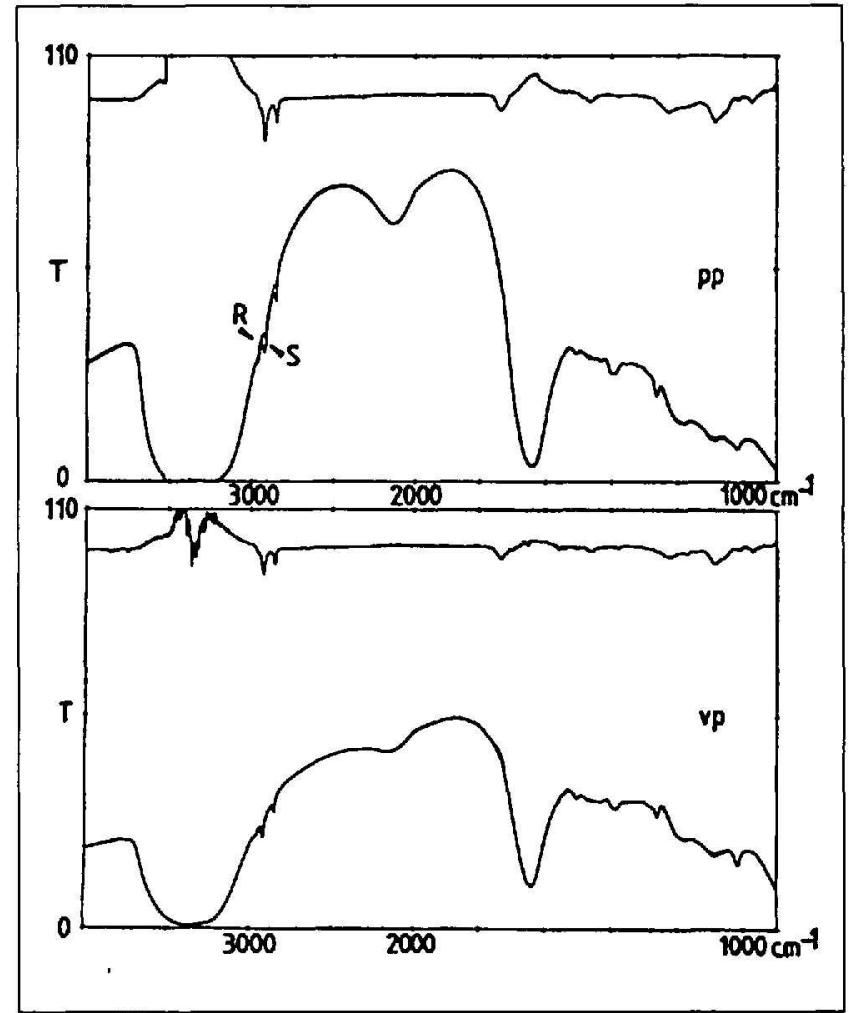

Fig. 5.PolarizedIR-ATR transmittance spectra of a palmitoyl-oleoyl-phosphatidylcholine (POPC) monolayer adsorbed tailltail to a DPPA $L B$ monolayer. The polar head groups of POPC are in contact with aqueous buffer solution (100 $\mathrm{mm} \mathrm{NaCl}, 20 \mathrm{~mm}$ phosphate, $\mathrm{pH} 7$ ). Angle of incidence: $\theta=45^{\circ}$, number of active internal reflections: $N=$ 30 , temperature: $T=20^{\circ}$. Reference: DPPA LB-monolayer, same buffer. From [62].

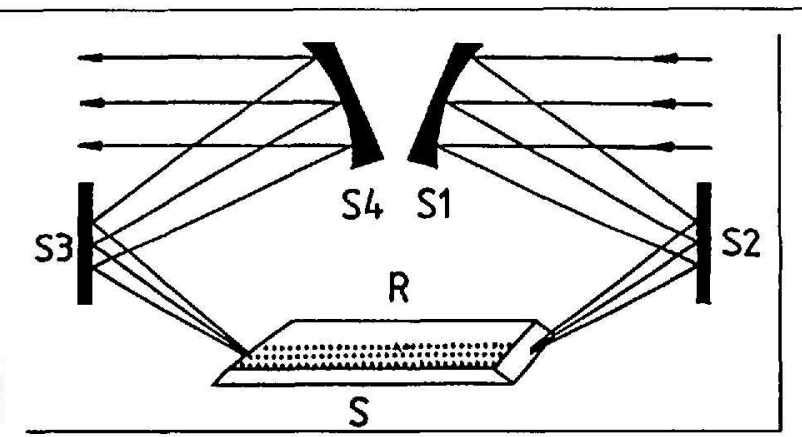

Fig. 6. Single-Beam-SampleReference (SBSR) attachment. The parallel incident beam is focused on the entrance face of a MIRE. The parallelism in the plate must be good to avoid crossing over between the sample $(S)$ and reference $(R)$ part. SI, S4: identical cylindrical mirrors. S2, S3: planar mirrors. The light is directed alternatively through the $S$ and $R$ side by means of a computer controlled chopper. From [62].

They decrease with decreasing time delay. SBSR technique, as described above, can reduce such instabilities considerably; however, best results are achieved in all cases in which modulation spectroscopy can be applied.

\subsubsection{Requirements for the Applicability of Modulation Spectroscopy}

The chemical or biological system to be investigated must allow a reversible or quasi-reversible periodic stimulation. If an external parameter is modulated by the circular frequency $\omega_{m}=2 \pi f_{m}$, all molecules of the sample which depend on this parameter will exhibit a concentration modulation of the same frequency, whereas all inert molecules remain unaffected. The use of phase-sensitive detectors (quadrature demodulation) enables a selective observation of the stimulated process in real time, whereas all inert components are suppressed (real time difference spectroscopy). Moreover, if the 'time constant' of the stimulated process is comparable to, or larger than the stimulation period, a phase lag and a decrease of the amplitude will result, which both are typical for the kinetics of the process.

\subsubsection{Theoretical Remarks .}

Consider a sample with concentration $c$, which may be influenced by a periodic modulation of an external parameter with circular frequency $\omega_{m}$. The resulting concentration modulation may then be described by the Fourier series Eqn. 31:

$$
\begin{aligned}
& c(t)=c_{\mathrm{o}}+2 c_{1} \sin \left(\omega_{m} t+\phi_{1}\right)+2 c_{2} \sin \left(2 \omega_{m} t\right. \\
& \left.+\phi_{2}\right)+\ldots=c_{\mathrm{o}}\left[1+\rho_{1} \sin \left(\omega_{m} t+\phi_{1}\right) "+\right. \\
& \left.\rho_{2} \sin \left(2 \omega_{m} t+\phi_{2}\right)+\ldots\right],
\end{aligned}
$$

where $c_{\mathrm{o}}$ denotes the mean concentration (DC term) and $2 c_{k}$ the corresponding Fourier coefficient of the $k \cdot \omega_{m}$ term. The degree of modulation is defined by

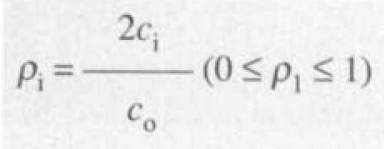


It should be noted that, if the stimulation is harmonic or rectangular, $\rho_{2}$ should only deviate from zero, if nonlinear reaction steps (other than first-order kinetics) occur in the chemical process.

According to Lambert-Beer's law, the light intensity $I_{\mathrm{s}}$ after passing the sample $S$ is given by

$$
I_{\mathrm{S}}=I_{0} \cdot T_{\mathrm{S}}=I_{0} \cdot 10^{-\varepsilon \varepsilon(t) d}=I_{0} \cdot 10^{-A_{S}}(33)
$$

where $T_{\mathrm{S}}$ and $A_{\mathrm{S}}$ denote transmittance and aborbance of the sample. Introducing Eqn. 31 into 33 , one obtains after reorganisation

$$
I_{\mathrm{S}}=I_{0} \cdot T_{\mathrm{S} 0} \cdot T_{\mathrm{S} 1} \cdot T_{\mathrm{S} 2} \cdot \ldots
$$

$T_{\mathrm{sk}}(k=1,2, \ldots)$ denotes the transmittance corresponding to the modulated resposes of the frequency $k \cdot \omega_{m}$.

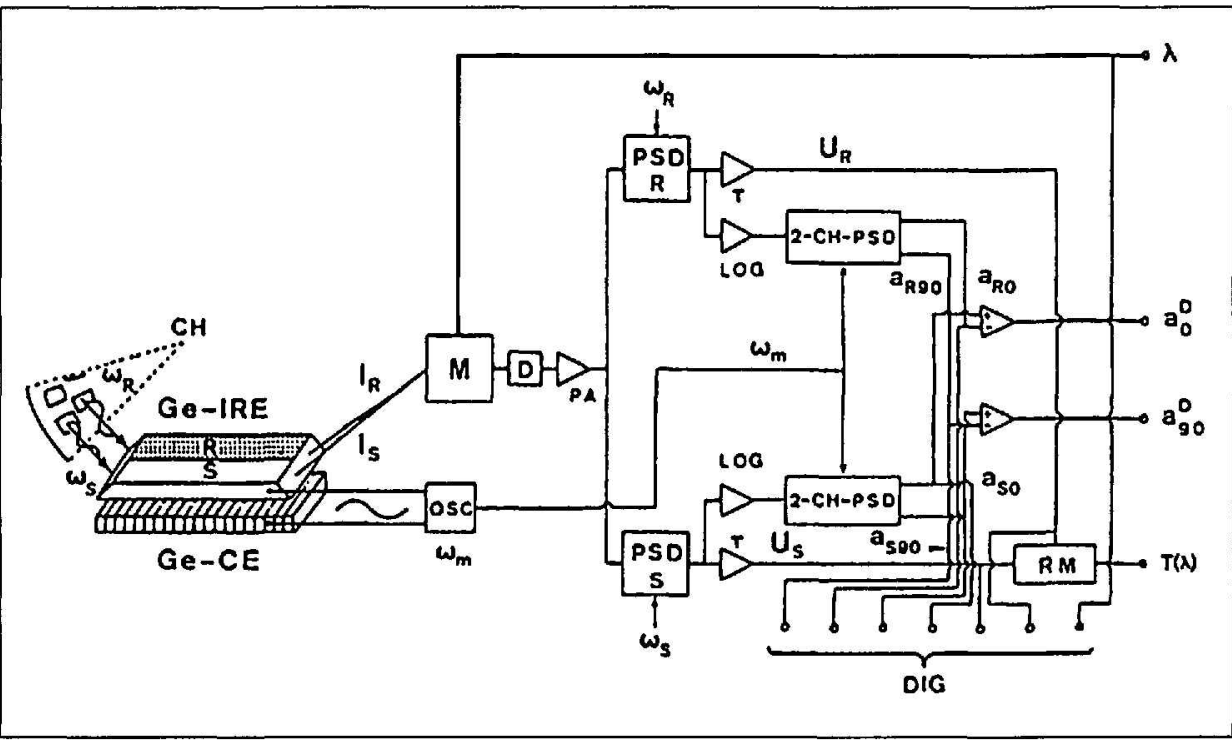

Fig. 7. Schematic set-up of a dispersive IR-ATR (SBSR) modulation spectrometer for electric-field stimulation [55][67][68]. $\mathrm{CH}$ : Chopper wheel with two rows of holes for modulation of incident light with circular frequencies $\omega_{\mathrm{S}}$ (sample), and $\omega_{\mathrm{R}}$ (reference); Ge-IRE: Ge internal reflection element with sample (S), and reference (R) area, see also Fig. 6. Ge-CE: Ge counter electrode for external electric-field stimulation with frequency $\omega_{m}$ by means of oscillator OSC. $I_{S}, I_{\mathrm{R}}$ intensities of sample, and reference beams, labelled by $\omega_{\mathrm{S}}$ and $\omega_{\mathrm{R}}$. M: Monochromator. D: Detector. PA: Preamplifier. PSD: Phase-sensitive detectors for demodulation of carrier frequencies $\omega_{\mathrm{S}}$ and $\omega_{\mathrm{R}}$. Stationary part. $\tau$. low pass filters, the electrical dc components $U_{\mathrm{S}}$ and $U_{\mathrm{R}}$ are fed to a ratiometer (RM), resulting in the transmittance spectrum $T(\lambda)$ of the sample. Dynamic part. LOG: logarithmic amplifier. 2-CH-PSD dual channel phase sensitive detector for the demodulation of externally stimulated $k \omega_{m}$ response $(k$ $=1,2, \ldots)$ of sample and reference $\left(a_{\mathrm{SO}}, a_{\mathrm{S} 90}, a_{\mathrm{R} 0}, a_{\mathrm{R} 90}\right)$ exhibiting $0^{\circ}$ and $90^{\circ}$ phase shift with respect to stimulation. $a_{0}^{\mathrm{D}}, a_{00}^{\mathrm{D}}$ : differencies between corresponding $0^{\circ}$ and $90^{\circ}$ components of sample and reference. $\lambda$ : wavelength. Relevant output signals are digitized (DIG) and stored in a computer.

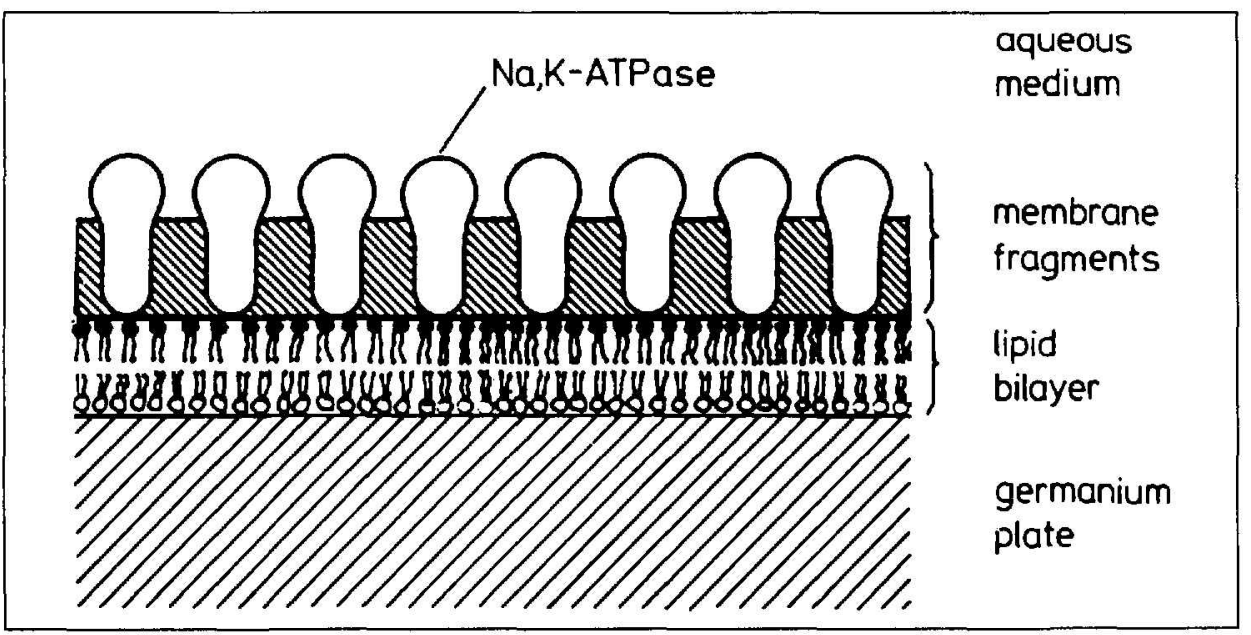

Fig. 8. Internal-reflection plate covered with lipid and protein layers. The Ge surface is initially coated with a phospholipid bilayer (see Sect. 4.I) and is in contact with aqueous solution. Replacing the aqueous solution is by a suspension of flat membrane fragments $0.2-1 \mu \mathrm{m}$ in diameter containing oriented $\mathrm{Na}^{+} / \mathrm{K}^{+}-$ATPase molecules with a density of several thousand per $\mu \mathrm{m}^{2}$, leads to binding of membrane fragments to the lipid bilayer. From [2].
A typical experimental set-up which enables the mesurement of modulation amplitudes $\rho_{k} \varepsilon c_{s 0}\left(\rho_{k}\right) d$, as well as the corresponding phase shifts $\phi_{k}$ with respect to the external stimulation is shown in Fig. 7. Both quantities, amplitude and phase shift, depend on the chemical reaction scheme and the rate constants. Therefore, reconstruction of a reaction scheme and determination of rate constants are possible by modulation spectroscopy at different stimulation frequencies $\omega_{m}$ [43][44][46].

\section{Applications to Drug/Membrane In- teraction Studies}

\subsection{Preparation of Immobilized Bilayer Membranes}

Model membranes used for drug interaction studies are phospholipid bilayers immobilized at the surface of the MIRE (Fig. 8).For technical reasons, the preparation must be performed in two independent steps [62]. The first monolayer is transferred by means of the LangmuirBlodgett technique [69]. The transfer from the air-water interface is achieved by withdrawing the ATR plate perpendicularly through the compressed monolayer at constant pressure and constant velocity (Fig. 9).

The monolayer coated dry plate is then mounted in a liquid sample cell which has been hydrodynamically optimized for flow-through experiments. For completion of the bilayer, a vesicular solution (I $\mathrm{mg}$ of lipid/ml of buffer, sonication under $\mathrm{N}_{2}$ flow at $\left.35-40^{\circ}\right)$ is circulated through the ATR cell (Fig . 10) resulting in spontaneous adsorption of phospholipid molecules.

The procedure was optimized based on electron micrographs (F.Kopp et al., to be published) leading to very stable and reproducible lipid bilayer membranes. No lipid loss was detectable by pumping a buffer solution in a closed cycle during 12 h $(1.9 \mathrm{ml} / \mathrm{min})$. Recently, corresponding mixed POPC/POPG bilayers have been prepared for melittin interaction studies [27].

\subsection{Preparation of Double Bilayer Assemblies}

It was shown earlier [72][73] that membrane fragments with enriched $\mathrm{Na}, \mathrm{K}$-ATPase isolated from rabbit kidney adsorb spontaneously to black lipid membranes (BLM) of POPC. It turned out that these membrane fragments adsorb to a DPPA/ POPC bilayer under the same conditions as they do to POPC BLM. The result is a double bilayer assembly as shown in Fig. 8 . This procedure offers new possibilities for in situ studies with integral membrane 
proteins in their 'natural' lipid environment. For details, see [2].

\subsection{Immobilization of Membranes, Proteins and Living Cells}

For dissolved substances ATR, spectroscopy requires minimum concentration of $c_{\text {min }} \approx 1-10 \mathrm{~mm}$, depending on the molar absorption coefficient. The sensitivity is drastically enhanced upon immobilization of the sample at the MIRE/water interface. Two possibilities have been described in Sect. 4.1 and 4.2. A variety of applications such as adsorption of lipid membranes, adsorption or chemical immobilization of proteins as well as living cells (e.g. erythrocytes) are enabled by silanization of the surface of a germanium ATR plate [67][74]. Aminopropyltriethoxysilane has turned out to be optimum as silanizing agent.

\subsection{Interaction of Drugs with Model Membranes}

Local anesthetics (LA) of the tertiary amine type (procaine (I), oxybuprocaine (II), falicaine (III), and dibucaine (IV)) were used as model compounds to interact with lipid bilayers of the type described in Sect. 4.1 (Formulae of LA).

All LAs were found to adsorb to the membrane. The higher the physiological activity of a given LA, the higher its affinity to condense to the DPPA/POPC bilayer, as well as to already adsorbed LA. Equilibrium surface concentrations have been determined and fitted by the Brunauer-Emmett-Teller (BET) isotherm (Fig. 11) [75].

From molecular-model considerations, one may estimate the maximum and minimum monolayer concentration of oxybuprocaine to be $\Gamma_{\max }=4.15 \cdot 10^{-6} \mathrm{~mol} / \mathrm{m}^{2}$ and $\Gamma_{\min }=1.38 \cdot 10^{-6} \mathrm{~mol} / \mathrm{m}^{2}$, respectively. Therefore, one has to conclude LAmultilayer condensation even at clinical application concentations (5-100 mM). More recent interaction studies of dibucaine (DIBU) with DPPA/POPC and DPPA/DMPC bilayers [61] have confirmed this finding. For these studies DIBU was dissolved $(0.5-10 \mathrm{~mm})$ in aqueous solutions of $100 \mathrm{~mm} \mathrm{NaCl}$ at $\mathrm{pH} 5.5$ (borate-phosphate-citrate buffer, $20 \mathrm{~mm}$ ) for DPPA/POPC membranes, and at $\mathrm{pH} 6.7$ (phosphate buffer, $20 \mathrm{~mm}$ ) with DPPA/ DMPC- $\mathrm{d}_{54}$ membranes. The volume of the ATR cell was $250 \mu \mathrm{l}$. To establish constant bulk concentration in the sample ATR cell, 5-10 ml of DIBU solution was permanently cirulated in a closed cycle, (Fig. 10).Fig. 12 shows typical polarized spectra of $10 \mathrm{~mm}$ DIBU in contact with a DPPA/POPC membrane (trace $a$ ). A corresponding membrane buffer solution was in the reference ( $R$ ) ATR cell, (see Fig. 6).

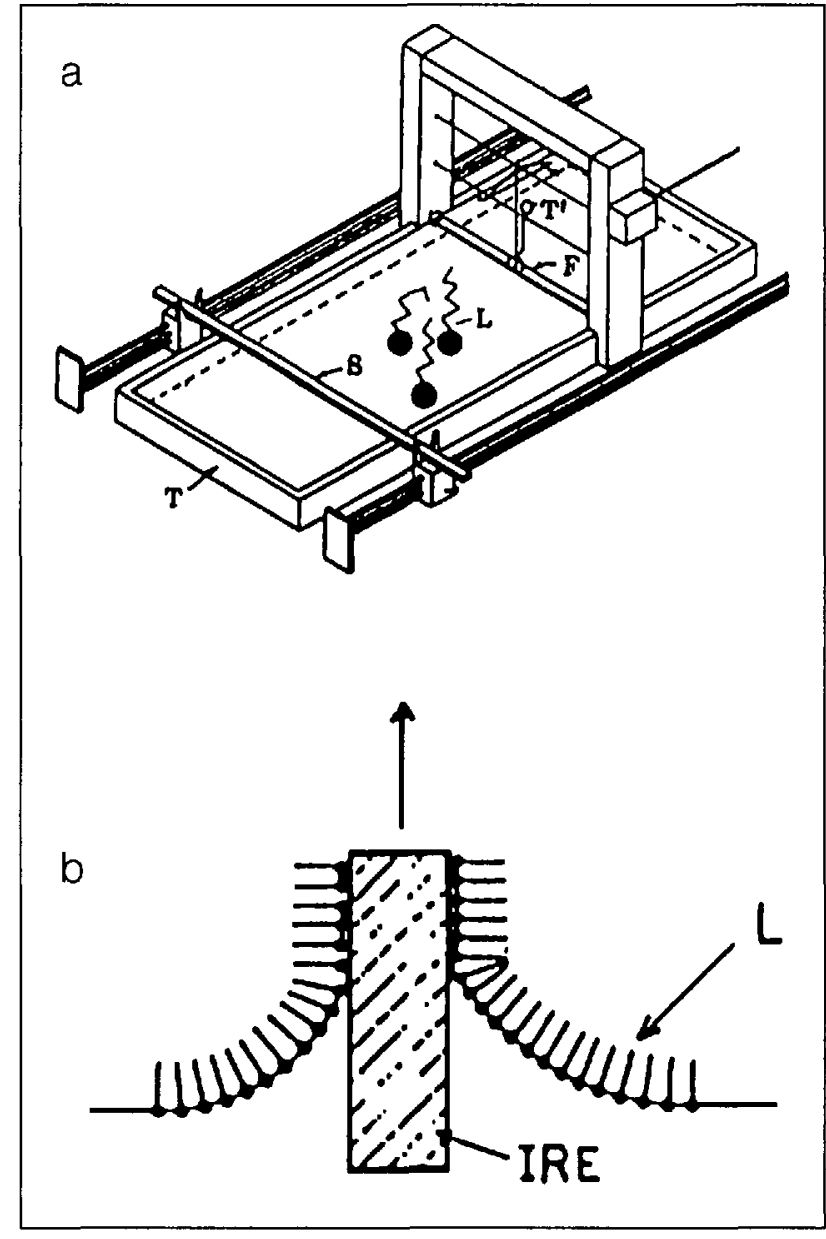

Fig. 9. a) Film balance. $T$, trough filled with aqueous subphase; $S$, sweep for compression of lipid monolayer (L) spread at the airwater interface; $F$, float connected to torsion wire ( $T$ ') for film pressure measurement. b) Transfer of a compressed lipid monolayer from the air-witer interface to a solid substrute (MIRE) by the Langmuir-Blodgett $t e c h$. nique, see [69-70].

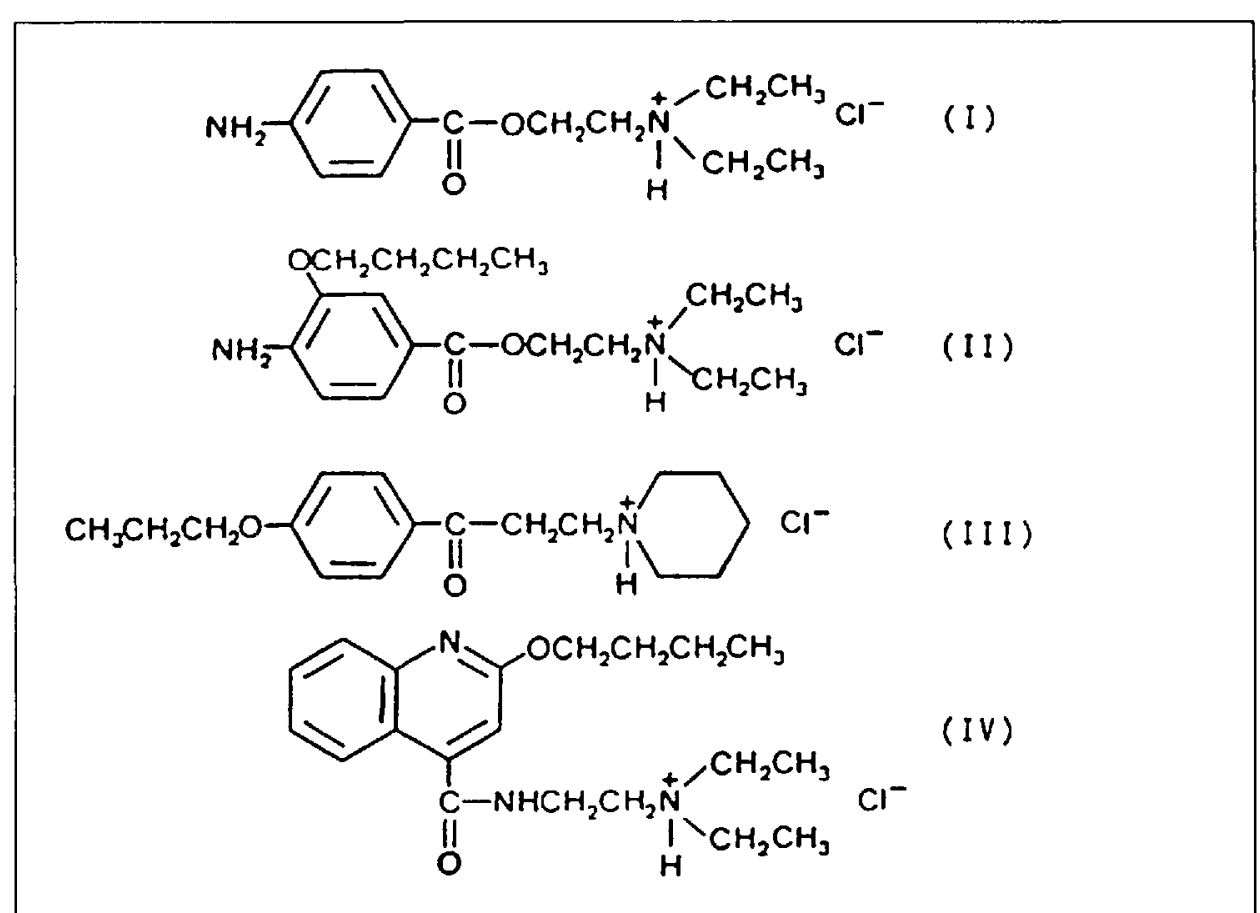

Trace $a$ of Fig. 12 reveals, therefore, membrane-bound and bulk DIBU, as well as DIBU-induced structural changes of the membrane. For comparison, corresponding spectra of 100-mm bulk DIBU were scaled $(x 0.37)$ to about the same intensity (trace $b$ ). Both experiments were performed at rather non-physiological pH 5.5 in order to enable direct comparison with published NMR data [76-79]. The fact that the absorbance spectrum of $100-\mathrm{mm}$ bulk DIBU has to be scaled by 0.37 instead of 0.10 in order to match the absorbance of $10-\mathrm{mm}$ DIBU interacting with the membrane, reveals unambiguously that about $2 / 3$ of the absorbance of DIBU results from membrane bound LA. Quantitative analysis by Eqns. 8,9, and 25-30 results in a surface concentration of total DIBU $\left(\mathrm{LA}_{\mathrm{tol}}\right)$ of $7.8 \cdot 10^{-6 \pm} 0.4 \cdot 10^{-6} \mathrm{~mol} / \mathrm{m}^{2}$. 


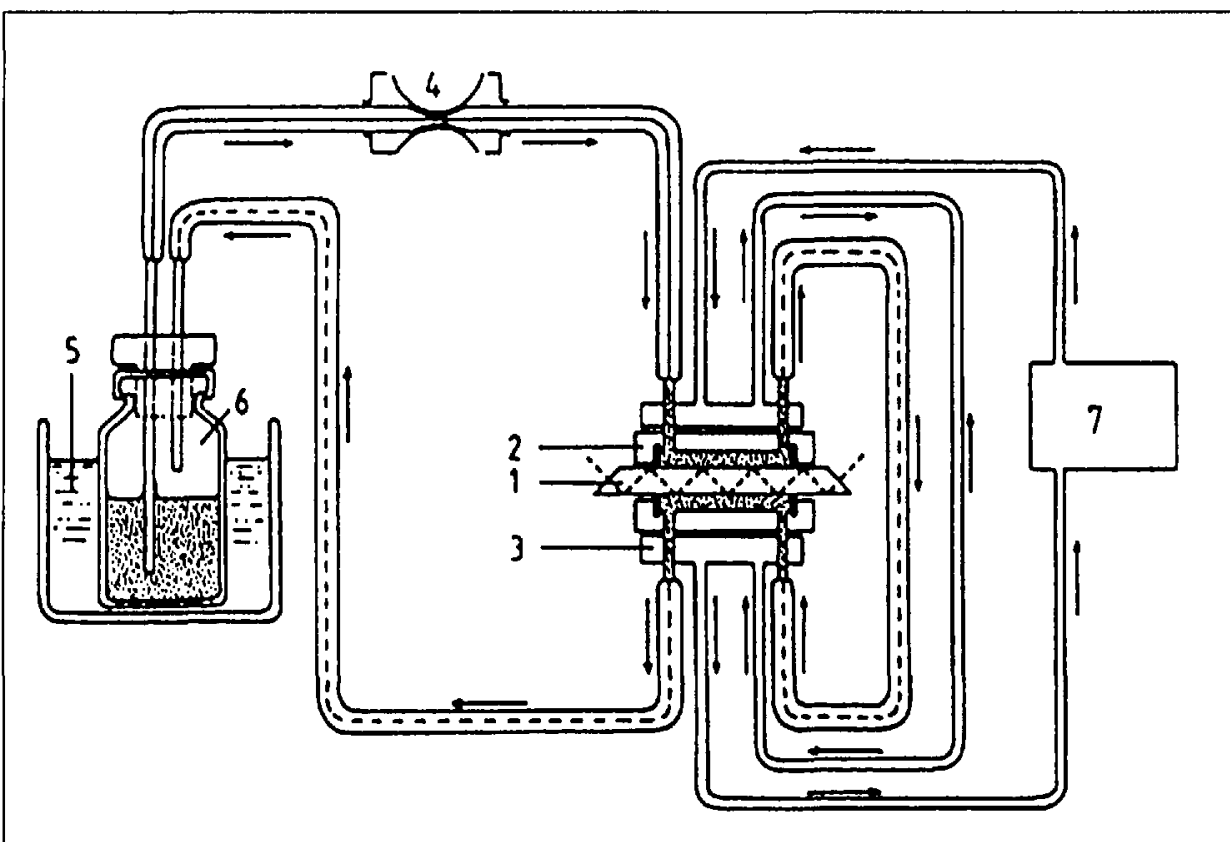

Fig. 10. Schematic set-up for IR-ATR in situ experiments. 1: Multiple intemal reflection element (MIRE, ATR plate), e.g. with immobilized membrane; 2: liquid sample cell for flow-through and stationary experiments; 3 : thermostat; 4: pump; 5 : thermostat for substrate reservoir; 6 : substrate reservoir; 7: MIRE thermostat. From [71].

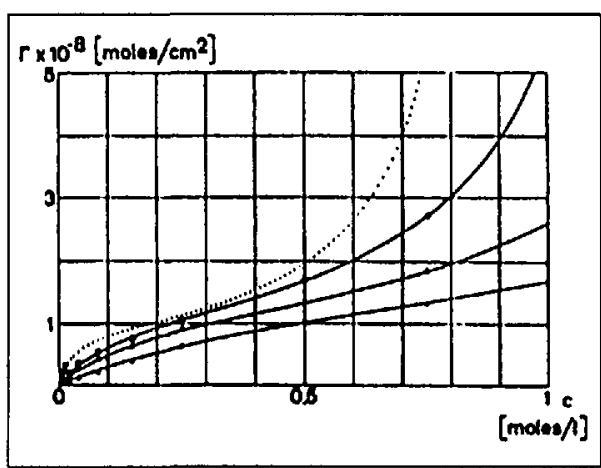

Fig. 11. Adsorption isotherms of procaine $(\bullet)$, lower trace, oxybuprocaine ( $\Delta)$, falicaine $(*)$, and dibucaine (o), upper trace. $20 \mathrm{~mm}$ phosphate buffer, $\mathrm{p} D 7.0,100 \mathrm{~mm} \mathrm{NaCl}$. Note, that increasing physiological LA activity is reflected by the increasing adsorption tendency. From [75].

Assuming a cross-section of $45-67 \mathrm{~A}^{2}$ per molecule [77], one obtains 2-3 DIBU monolayers bound to the DPPA/POPC membrane at $10-\mathrm{mm}$ bulk concentration. In contrast, Seelig et al. [77], reported still sub-monolayer coverage at the same bulk

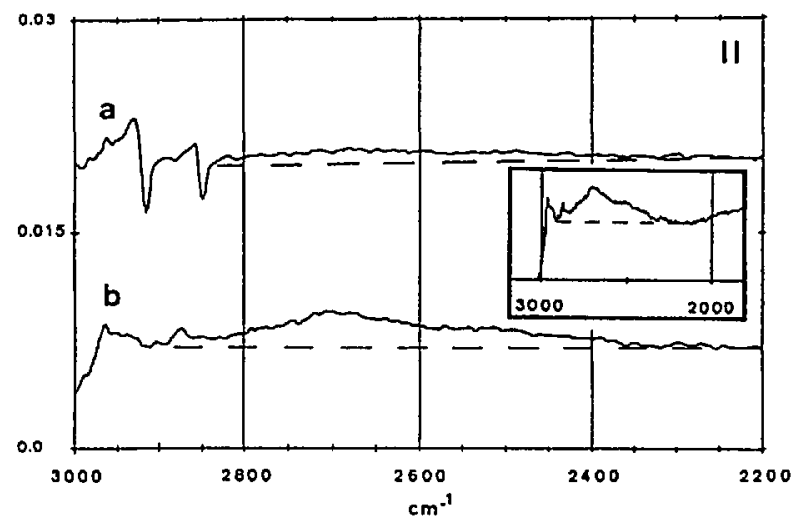

A

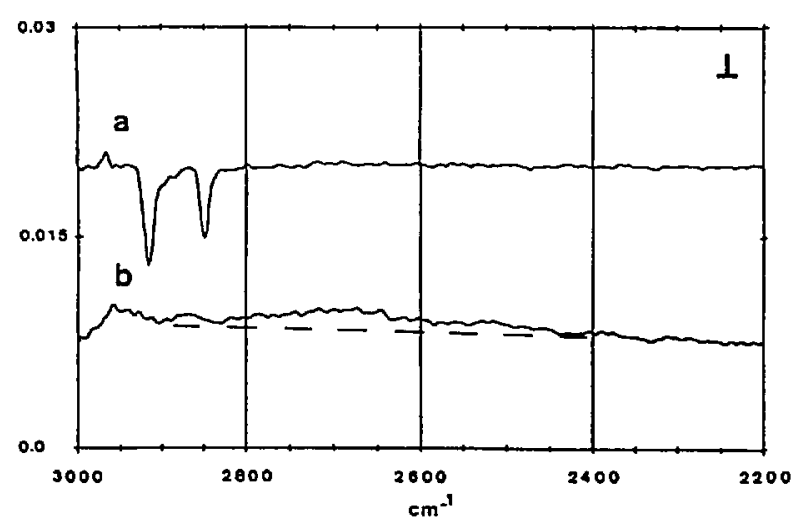

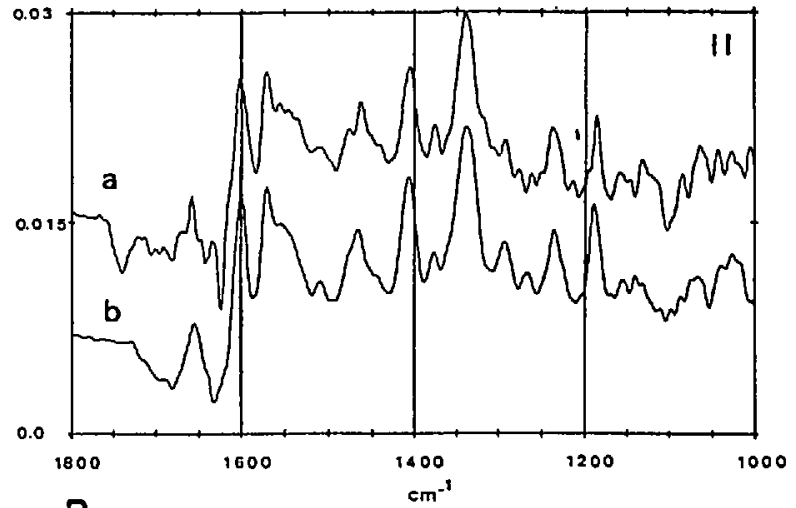

B

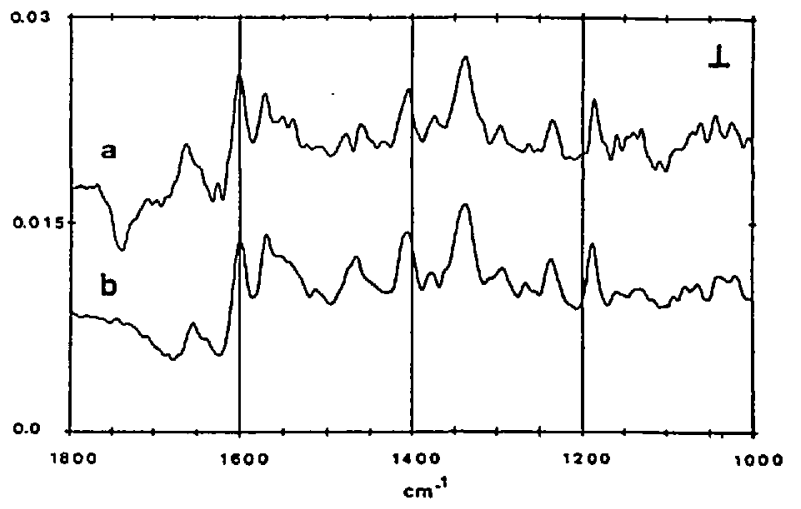

Fig. 12. Polarized IR-ATR absorbance spectra of a 10 -mM dibucaine (DIBU) solution (100 mM NaCl, $20 \mathrm{~mm}$ borate-phosphate-citrate (BPC) buffer, $\mathrm{pH}$ 5.5) interacting with a DPPA/POPC bilayer membrane (trace a), and for comparison, 100-mM DIBU solution in contact with the pure Ge $A T R$ plate (trace b), scaled with 0.37 in order to get approximately the same band intensities (same buffer). Insert: Compressed wavenumber scale of the latter in the $v\left(\mathrm{NH}^{+}\right)$ region (same absorbance scale). Angle of incidence: $\theta=45^{\circ}$, number of active internal reflections: $N=19$, temperature: $T=25^{\circ}$. Reference: DPPA/POPC bilayer (see Sect. 4.1), same buffer. Wavenumber range: $3000-2200 \mathrm{~cm}^{-1}(\mathrm{~A}), 1800-1000 \mathrm{~cm}^{-1}(\mathrm{~B})$. Note. $i$ ) Only ca. $1 / 3$ of the DIBU absorbance (trace a) results form dissolved DIBU. $2 / 3$ is bound DIBU as a consequence of the scaling factor 0.37 for the trace $b$ spectrum. Calculated surface concentration: $\Gamma=(7.8 \pm 04) 10^{-6} \mathrm{~mol} \mathrm{~m}^{-2}$. ii) Dissolved DIBU is protonated $\left(\mathrm{p} K_{\mathrm{a}}=8.83\right)$. Membrane-bound DIBU appears partly as base, as concluded from corresponding $v\left(\mathrm{NH}^{+}\right)$absorbance near $2700 \mathrm{~cm}^{-1}$. iii) The unusual behaviour of typical lipid bands $(v(\mathrm{CH}), v(\mathrm{C}=0), v(\mathrm{C}-\mathrm{O}), v(\mathrm{POC}))$ is reversible and does not reflect lipid loss, see text. 
DIBU concentration and $\mathrm{pH}$. One reason for the discrepancy results probably from different bilayers. Symmetric POPC multilayers were used in [77]. In our case, also POPC was faceing the aqueous environment (DIBU), however, the counter monolayer, faceing the Ge-ATR plate, was $\mathrm{Ca}^{++}$DPPA. Although the DPPA polar region is not directly accessible by DIBU (Fig. 8), the resulting negative trans-membrane electric field could facilitate DIBU binding and incorporation into the membrane. A second reason could be of systematic nature, since the experimental approaches are quite different (see Sect.4.5).

Concerning the lipid response to DIBU binding, there is evidence for a reorientation of the choline head group (negative band at $1491 \mathrm{~cm}^{-1}$ [45]) in agreement with [77][80]. More significant lipid responses, however, result from $v\left(\mathrm{CH}_{2}\right): 2800$ $3000 \mathrm{~cm}^{-1}, v\left(\mathrm{CD}_{2}\right): 2000-2300 \mathrm{~cm}^{-1}$, $v(\mathrm{C}=\mathrm{O}):-1740 \mathrm{~cm}^{-1}, \delta\left(\mathrm{CH}_{2}\right): 1470 \mathrm{~cm}^{-1}$, $v(\mathrm{C}-\mathrm{O}): \sim 1180 \mathrm{~cm}^{-1}, v_{s}\left(\mathrm{PO}_{2}^{-}\right)$and for $v_{\mathrm{as}}\left(\mathrm{PO}_{3}^{-}\right): \sim 1100 \mathrm{~cm}^{-1}, v_{s}\left(\mathrm{PO}_{3}^{-}\right): \sim 1000$ $\mathrm{cm}^{-1}$.

Since the reference to the spectra shown in Figs. 12 and 13 are the corresponding bilayer membranes and buffer solutions the negative responses of lipid bands could indicate material loss upon LA interaction. This, however, is not the case, because the effects are reversible after LA replacement by buffer [61].

A similar, also less pronounced behaviour was also observed earlier with the LA oxybuprocaine [62]. LA-induced conformational changes in the hydrocarbon chain, fatty-acid ester, phosphate, and choline regions have to be concluded. Hydrocarbon-chain disordering is also indicated by broadening and upwards shifts of $v_{\mathrm{ass}, \mathrm{s}}\left(\mathrm{CH}_{2}\right)$ and $v_{\mathrm{as}, \mathrm{s}}\left(\mathrm{CD}_{2}\right)$ [61]. Both phenomena are qualitative features for the formation of gauche defects [81]. Quantitative analysis is possible via the dichroic ratio dependence of $v_{\text {ss } s}\left(\mathrm{CH}_{2}\right)$ on bulk DIBU concentration (Fig. 14), or by dichroic analysis of the difference bands ( $F$ igs. 12 and 13). Concerning the former, Fig. 14 shows the dependence of the mean dichroic ratio of $v_{\mathrm{as}}\left(\mathrm{CH}_{2}\right)$ and $v_{\mathrm{s}}\left(\mathrm{CH}_{2}\right)$ of a DPPA/POPC membrane interacting with DIBU (S) at $\mathrm{pH} 5.5$ and with buffer (R).

Since data were collected in the SBSR mode (see Fig. 6) the age of both membranes, $S$ and $R$, was exactly the same. The $v\left(\mathrm{CH}_{2}\right)$ dichroic ratio of the $\mathrm{R}$ membrane remained constant over the whole duration of the experiment. Maximum dichroic ratio $(1.37 \pm 0.04)$ is reached at $c a .3-\mathrm{mm}$ bulk concentration, indicating saturation of the effect. This could mean that maximum chain disordering is reached. Since 3-mm bulk DIBU correspond to more than a monolayer adsorbed DIBU [61], and

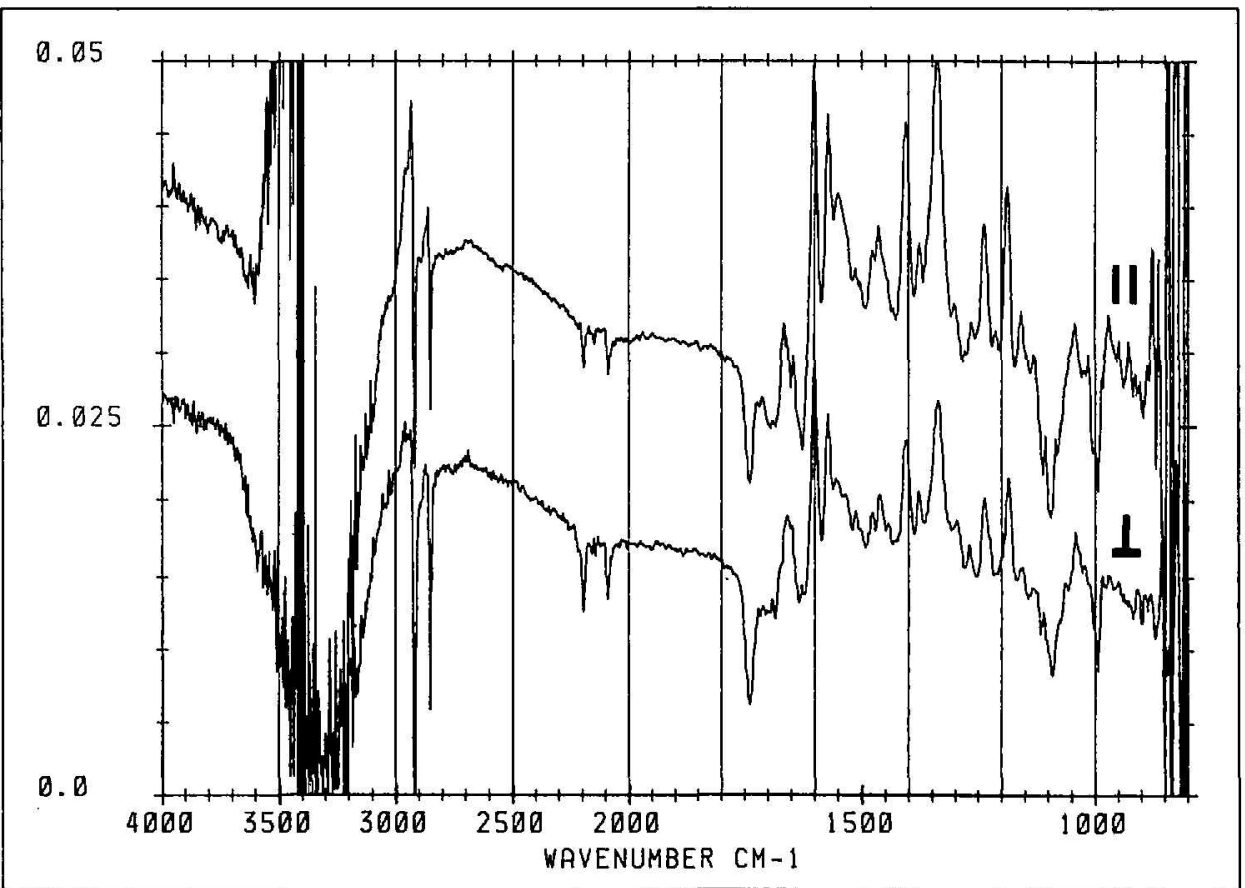

Fig. 13. Polarized IR-ATR absorbance spectra of a $10-\mathrm{mm}$ dibucaine $(D I B U)$ solution $(100 \mathrm{~mm} \mathrm{NaCl}$, $20 \mathrm{~mm}$ phosphate, $\mathrm{pH} 6.7$ ) interacting with a DPPAIDMPC-d ${ }_{54}$ bilayer membrane. Angle of incidence: $\theta=45^{\circ}$, number of active internal reflections: $N=26$, temperature: $T=25^{\circ}$. Reference: DPPA/DMPC- $\mathrm{d}_{54}$ in contact with the same buffer. Note. The response of the hydrocarbon chains $v(\mathrm{CH}): 2800-3000 \mathrm{~cm}^{-1}, v(\mathrm{CD}): 2100-2300 \mathrm{~cm}^{-1}$, the fatty acid ester groups $v(\mathrm{C}=\mathrm{O}): 1740 \mathrm{~cm}^{-1}$, $v(\mathrm{C}-\mathrm{O}): 1180 \mathrm{~cm}^{-1}$, and $v_{\mathrm{s}}\left(\mathrm{PO}_{2}^{-}\right), v_{\mathrm{as}}\left(\mathrm{PO}_{3}^{-}\right): 1100 \mathrm{~cm}^{-1}, v_{\mathrm{s}}\left(\mathrm{PO}_{3}^{-}\right): 1000 \mathrm{~cm}^{-1}$.

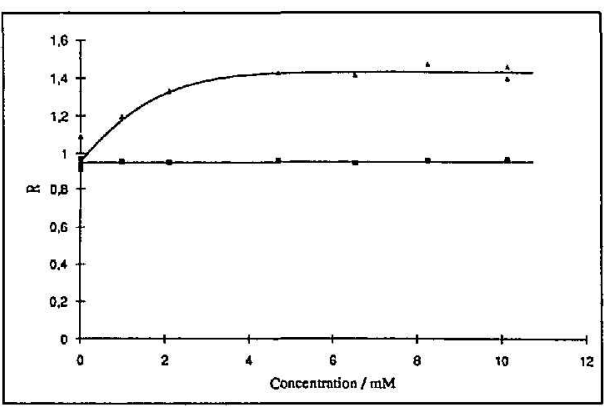

Fig. 14. Dependence of the mean dichroic ratio of symmetric and antisymmetric $\mathrm{CH}_{2}$ stretching of a DPPAIPOPC bilayer on bulk DIBU concentration. $100 \mathrm{~mm} \mathrm{NaCl}, \mathrm{BPC}$ buffer $\mathrm{pH} 5.5,25^{\circ} . \mathrm{S}$ : Sample, 0-10 mм DIBU. R: Reference, same as $S$ except without DIBU.

since an isotropic layer would have a dichroic ratio of $R_{\text {iso }}=1.75 \pm 0.05$, it rather tends to the interpretation, that DIBU coats the membrane, thus, preventing further disordering. On removing the $10-\mathrm{mm}$ DIBU solution, a certain hydrocarbonchain disordering is restored, although no remaining DIBU could be detected in the membrane. These data, and the directly measured difference spectra (Figs. 12, and 13) enable the determination $\sum_{i, k} \sigma_{i, k}^{\mathrm{S}}, \sum_{i, k} \sigma_{i, k}^{R}$, and $\sum_{i, k}\left(\sigma_{i, k}^{S}-\sigma_{i, k}^{R}\right)$, respectively. $\sigma_{i, k}$ denotes the orientation function (Eqn. 21$)$ of the k-th $\mathrm{CH}_{2}$ group in the $i$-th chain ( $i=$ 1.2) (to be published). Preliminary simulations by Eqn. 23 reveal that the magnitude of the change of dichroic ratios under the influence of DIBU (Fig. 14) as well as the prominent $v\left(\mathrm{CH}_{2}\right)$ response (Fig. 13) requires disordering of the hydrocarbon chains of the inner DPPA monolayer, too. The preliminary assignment of the difference band at $1000 \mathrm{~cm}^{-1}$ (Fig. 13) to $v_{\mathrm{s}}\left(\mathrm{PO}_{3}^{-}\right)$gives some evidence for DIBU permeation across the membrane.

Unambiguous information on conformation and orientation of bound DIBU would only be available from partly isotopic labelled LA, e.g. ${ }^{18} \mathrm{O}$ and ${ }^{2} \mathrm{H}$ in oxybutyl, ${ }^{15} \mathrm{~N}$ selectively in the aromatic ring, amide, and ammonium group. Nevertheless, a preliminary analysis of actual data is given in [61].

\subsection{Drug-Membrane Partition Coeffi- cient}

Since the volume of a bilayer membrane is small and in some cases may become comparable to that of bound drug, it is reasonable to define the apparent partition coefficient by

$$
P_{\text {app }}=\lim _{c_{b} \rightarrow 0} \frac{c_{m}}{c_{b}}
$$

where $c_{m}$ and $c_{b}$ denote the drug concentrations in the membrane and in the bulk [82]. A number of different methods for partition coefficent determination have been applied so far. The most common one is equilibration of the drug with lipid vesicles, followed by centrifugation and determination of the drug in the supernatant by optical methods. The membrane bound amount is then calculated by via mass conservation. Pellets are often used for further investigations by NMR or ESR 

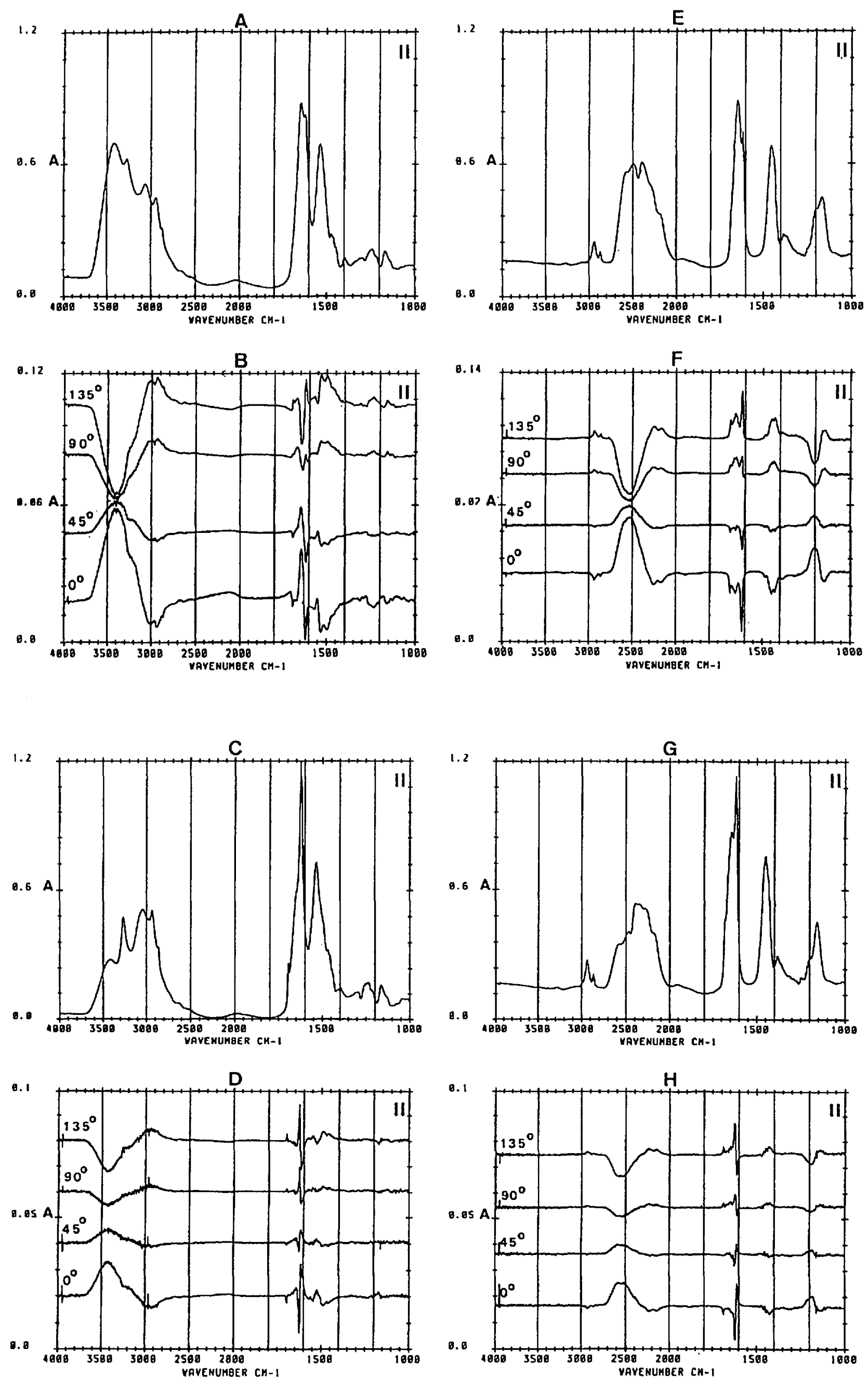
Fig. 15. Stationary and temperature $(\mathrm{T})$ modulated FTIR ATR spectra of hydrated poly(L-lysine) multilayers on a Ge ATR plate (MIRE). Environment: $\approx 90 \%$ rel. humidity $\mathrm{H}_{2} \mathrm{O}(\mathrm{A}-\mathrm{D})$ and $\mathrm{D}_{2} \mathrm{O}$ (E-H). Mean temperatures: $T=26^{\circ}$ (A,B,E,F) and $T=35^{\circ}(\mathrm{C}, \mathrm{D}, \mathrm{G}, \mathrm{H})$. Stationary spectra: Predominant antiparallel $\beta$ structure (pleated sheet) at $35^{\circ}\left(1625 / 1695 \mathrm{~cm}^{-1}, \mathrm{C}\right.$, and $1618 / 1692 \mathrm{~cm}^{-1}$, G). More complex conformational equilibrium at $26^{\circ}, \alpha$-helix and/or random $\left(1655 \mathrm{~cm}^{-1}, \mathrm{~A}, 1643\right.$ $\mathrm{cm}^{-1}, E$ ) in equilibrium with antiparallel $\beta$ structure. Amide I band in $\mathrm{H}_{2} \mathrm{O}$ environment overlapped by $\delta\left(\mathrm{H}_{2} \mathrm{O}\right)\left(\sim 1640 \mathrm{~cm}^{-1}, A, C\right)$. Complete $\mathrm{H} / \mathrm{D}$-exchange in $\mathrm{D}_{2}$ Oenvironment $(E, G, v(\mathrm{NH})$ : $\sim 3300 \mathrm{~cm}^{-1}, v(\mathrm{ND}): \sim 2400 \mathrm{~cm}^{-1}$, amide II: $\sim 1540 \mathrm{~cm}^{-1}$, amide II': $\sim 1450 \mathrm{~cm}^{-1}$ ). T-modulation spectra: amplitude: $\Delta T= \pm 1.5^{\circ}(B, F), \Delta T=$ $\pm 1.0^{\circ}(D, H)$. Period: $\tau_{m}=11 \mathrm{~min}($ i.e. $5.5 \mathrm{~min} T$ $\Delta T$ and $5.5 \mathrm{~min} T+\Delta T$ ). Phase shift with respect to stimulation: $0^{\circ}$ (lower trace), $45^{\circ}, 90^{\circ}, 135^{\circ}$ (upper trace). Modulation bands of $\mathrm{H}_{2} \mathrm{O}(\sim 3400$ $\left.\mathrm{cm}^{-1}, \sim 1640 \mathrm{~cm}^{-1}\right)$ and $\mathrm{D}_{2} \mathrm{O}\left(\sim 2500 \mathrm{~cm}^{-1}, \sim 1200\right.$ $\mathrm{cm}^{-1}$ ) result from temperature- and conformation-induced hydration changes, therefore, amide $\mathrm{I}\left(\sim 1650 \mathrm{~cm}^{-1}\right)$ analysis is more relevant in $\mathrm{D}_{2} \mathrm{O}$ environment $(F, H)$. Modulation at $T=35^{\circ} \mathrm{stim}$ ulates a transition $\left(1625 / 1619 \mathrm{~cm}^{-1}, D, 1620 /\right.$ $1616 \mathrm{~cm}^{-1}, H$ ) probably between two $\beta$ structures. This transition occurs at $T=26^{\circ}$, too $\left(90^{\circ}\right.$ phase shift, 3rd trace from bottom, $B, F)$. However, $\alpha$ helices and/or random structures are also involved $\left(\sim 1655 \mathrm{~cm}^{-1}\right)$ The complexity of the transition is reflected by the phase dependent shapes of the amide I,I' bands, which is typical for multistep reactions. Angle of incidence: $45^{\circ}$ number of active internal reflections: $N=26$. Reference for stationary spectra: Clean MIRE.

[76-79][83-86]. Other methods are based on dialization [87], freezing-point depression [88][89], and electrophoretic mobility $(\zeta$-potential) measurements [83][90]. Since many drugs may assume a charged and uncharged state (amines, carboxylic acids) depending on the environment, it is reasonable to take electrostatic effects into account for the discussion of membrane partitioning. Most of these approaches are based on the Gouy-Chapman model [77][83][88][91-94]. There is general agreement that tertiary amines (e.g. LAs) undergo an apparent $\mathrm{p} K_{\mathrm{a}}$ decrease of $\Delta \mathrm{p} K_{\mathrm{a}}$ $\approx 1.5$ upon membrane binding. Membrane bound LAs with a bulk $\mathrm{p} K_{\mathrm{a}}$ between 8 and 9 may, therefore, be expected predominantely in the protonated state at bulk $\mathrm{pH}$ 5.5 [76-79], which is in contrast to our finding, see below.

Although these methods are generally applied, there is still room for some critical remarks. Firstly, concerning the centifugation approach: to get a supernatent free of lipids, up $300000 \mathrm{~g}$ are applied for $1-3 \mathrm{~h}$. This leads probably to an equilibrium between drug in the supernatant, in the interlamellar water phase (the pellet consists of multibilayers), and in the mem-

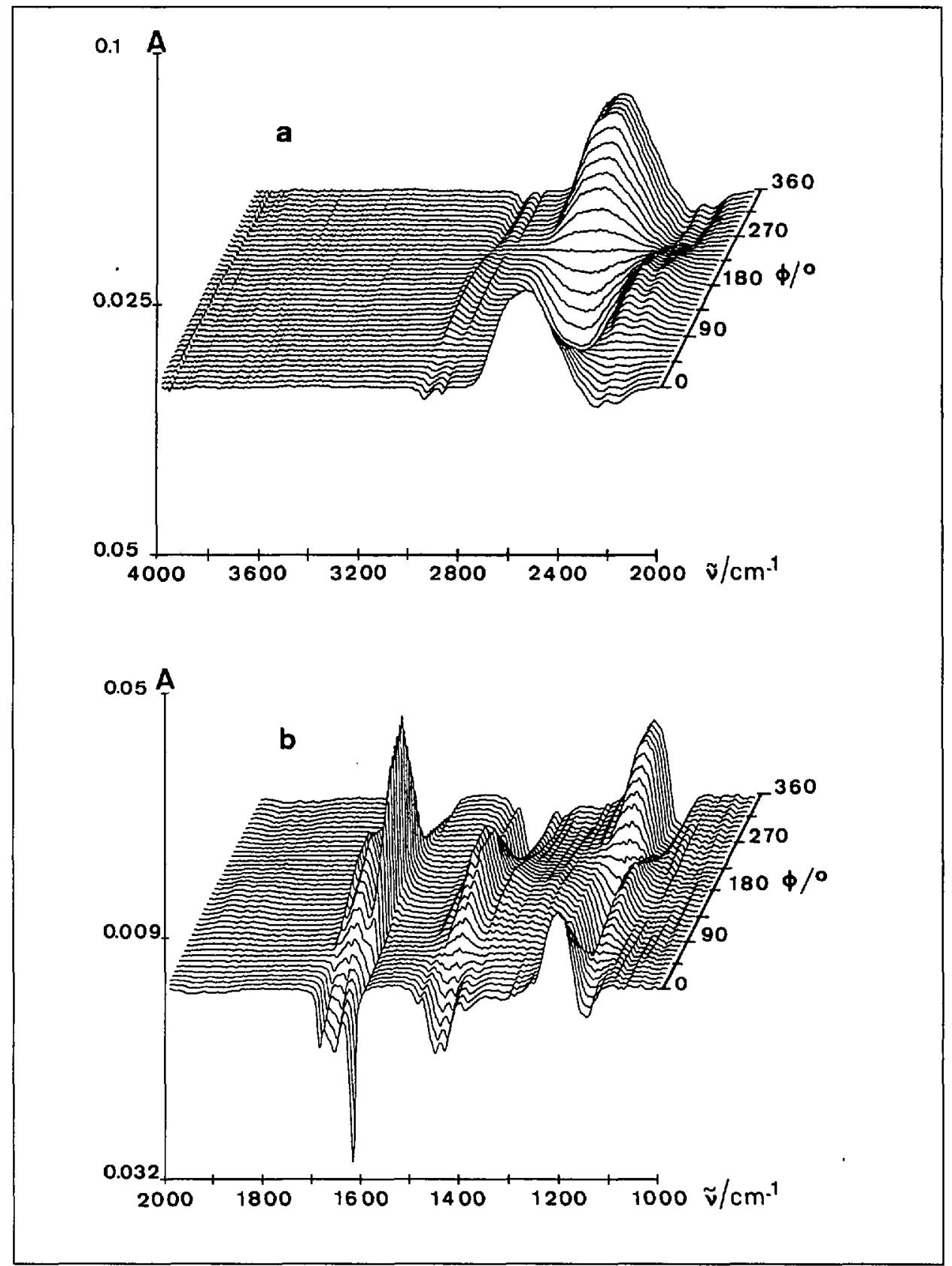

Fig. 16. Temperature-(T) modulated FTIR ATR spectra of $\mathrm{D}_{2} \mathrm{O}$ hydrated poly (L-lysine) multilayers. The spectra result from the same experiment as described in the legend to $F$ ig. $15 F$. Phase resolution $9^{\circ}$. Phase delay with respect to $T$ stimulation: $0^{\circ}$ first spectrum (foreground), $351^{\circ}$ last spectrum. $a$ ) $\left.4000-2000 \mathrm{~cm}^{-1}, b\right) 2000-1000 \mathrm{~cm}^{-1}$.

brane. One may ask, whether this system at the moment of separation of supernatant and pellet still represents the equilibrium state before centrifugation? From the thermodynamic point of view, it is not only a question of high-pressure application, but also of activity alteration in the interbilayer water phase. Secondly, many questions arise with respect to the electrostatic approach: is the Gouy-Chapman model adequate to describe the inhomogeneous surface potential profile resulting from single adsorbed charges? In this respect, an alternative approach has been given by Ninham and Parsegian [95] taking account of the local potential of membranebound charged species. Furthermore, can counter-ion binding be neglected as in most applications cited above? Why is the interfacial and bulk permittivity set to be equal, although considerable differences have to be expected [96]? This has influence on the Boltzmann distribution of ions between bulk and interface and results in a Born energy term. Concerning $\zeta$-potential measurements, there are also uncertainties with respect to non-uniform vesicle population, distance of the plane of shear from the vesicle surface (generally assumed to $d_{\zeta}=2 \AA$ ). Alteration of surface structure by the adsorption of charged molecules may also influence $d$.

Finally, adsorption of charged partiinduce compensating reactions such as counter-ion adsorption and dissociation or association of $\mathrm{H}^{+}\left(\mathrm{p} K_{\mathrm{a}}\right.$ shift $)$. Structural alterations may occur, too. cles leads to a surface potential, which will 
One should be aware, that in cited applications many 'hidden' parameters had to be estimated in order to enable a quantitative analysis of experimental data. The need for complementary experimental techniques with direct access to the quantities of interest is obvious. In this respect, IR ATR spectroscopy is a promising approach. Typically, the drug solution is circulated through the sample cell (see Figs. 6 and 10). Therefore, there is no ambiguity with respect to the bulk concentration. If necessary, it can be monitored on line. On the other hand, drug binding is detected simultaneously in situ via ATR. Quantitative analysis is straightforward as described in Sect. 2.

Concerning DIBU binding to a DPPA/ POPC membrane, we have measured the adsorption isotherms for both, total DIBU $\left(\mathrm{LA}{ }_{\text {tot }}\right)$ using the aromatic ring vibration at $1407 \mathrm{~cm}^{-1}$, and protonated species $\left(\mathrm{LAH}^{+}\right)$ by means of the $\mathrm{N}-\mathrm{H}^{+}$stretching vibration at $2700 \mathrm{~cm}^{-1}$ [61]. It follows that, already at bulk DIBU concentration of $c a .1 \mathrm{mM}$, the bound total amount of DIBU is sufficient to form a compact monolayer. However, the bound protonated species $\mathrm{LAH}^{+}$ reaches this limit only at 2-6-mm bulk concentration, although there is $99.96 \%$ $\mathrm{LAH}^{+}$and only $0.04 \% \mathrm{LA}$ at $\mathrm{pH} 5.5$ in the bulk environment $(\mathrm{p} K=8.83)$. Furthermore, about one half of bound DIBU is deprotonated and exists as base (LA) even at bulk $\mathrm{pH}$ 5.5. This ratio of charged to uncharged DIBU is also maintained in the DIBU multilayer region at $\geq 5 \mathrm{~mm}$ bulk concentration. Note that Fig. 12 gives already evidence for significant DIBU deprotonation upon binding to the membrane. It should be mentioned that the bulk concentration of $\mathrm{LA}^{2}$ and $\mathrm{LAH}^{+}$are known and maintained constant during the adsorption process by using a closed cycle flow-through system (Fig. 10) with a large buffered substance reservoir. The adsorption isotherms of $\mathrm{LAH}^{+}$and $\left(\mathrm{LA}_{\mathrm{tot}}\right)$ fit well to the simple Langmuir isotherm $\Gamma=$ $\mathrm{c}_{\mathrm{b}} \mathrm{B} /\left(a+c_{\mathrm{b}}\right) . B$ and $a$ denote the monolayer surface concentration, and the ratio of desorption/adsorption rate constants. The concentration of a compound in the membrane $c_{m}$ is related to the experimentally accessible surface concentration $\Gamma$ by $c_{m}$ $=\Gamma / d_{0}$, where $d_{\mathrm{o}}$ denotes the membrane thickness $\left(d_{0}=5 \mathrm{~nm}\right)$. According to $E q n$. 35 , the apparent partition coefficient may be expressed by the Langmuir parameters and the membrane thickness

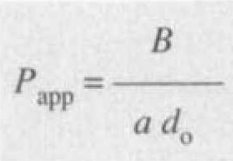

The resulting partition coefficients are found to be $P_{\mathrm{app}}\left(\mathrm{LA}_{\mathrm{tot}}\right)=1060 \pm 86$,
$P_{\mathrm{app}}\left(\mathrm{LAH}^{+}\right)=475 \pm 93$, and $P_{\mathrm{app}}(\mathrm{LA})=$ $9.99 \cdot 10^{5} \pm 1.87 \cdot 10^{5}$, all data with $95 \%$ confidence limits [61]. The values for the total amount $\mathrm{LA}_{\text {tot }}$ and for $\mathrm{LAH}^{+}$are experimental, whereas values for the base LA are calculated from the difference of the surface concentrations of $\mathrm{LA}_{\text {tot }}$ and $\mathrm{LAH}^{+}$. The Langmuir parameters $B$ and a lose their original meaning and have to be considered as fitting parameters for an experimental isotherm, since electrostatic interactions as well as surface reactions $\left(\mathrm{LAH}^{+} \rightarrow \mathrm{LA}+\mathrm{H}^{+}\right)$and multilayer adsorption occur. In view of the finding that considerable amounts of DIBU base exist, probably in the hydrophobic region of the membrane, electrostatic approachs on the basis of the assumption that every bound DIBU carries a positive charge to the membrane surface are not reasonable, even at bulk $\mathrm{pH}=5.5$. An interfacial $\mathrm{p} K_{\mathrm{a}}$ shift of ca. -1.5 units may still be true (although there is poor experimental substanciation, see above), since DIBU base can escape from the interface into more hydrophobic membrane regions. Ellipsometry could give valuable additional information on membrane thickness during the adsorption process, as well as on surface roughness and optical constants.

\subsection{Modulation Spectroscopy}

Modulation spectroscopy is a very sensitive and selective technique for the detection of weak periodic changes of the state of a sample induced by an external periodic change of any thermodynamic parameter. Moreover, detailed kinetic information is available, too. Modulation techniques have a wide field of applications [97] (see also Sect. 3.4) because of considerable signal/noise ( $\mathrm{S} / \mathrm{N}$ ) enhancement with respect to stationary procedures. In our case, we estimate a $\mathrm{S} / \mathrm{N}$ enhancement by a factor of about 10 .

Polarization modulation $(\sim 50 \mathrm{kHz})$ was the first application with FTIR instruments [98]. This method, however, is of minor importance in bio FTIR spectroscopy. In conjunction with ATR it is even inadequate because of different effective thicknesses for $\|$-, and $\perp$-polarized incident light, (see Eqns. 8-10), and 'Dichrioic Difference Spectra' (Sect. 2.3.5).

Here, we report the first FTIR modulation spectra at low stimulation frequency [99]. Temperature $(T)$ modulation of hydrated poly $(L$-lysine $)$ was used, because it exhibits a conformational equilibrium which is very sensitive to hydration and $T$ changes as well as to $\mathrm{pH}$ changes [100105]. $T$-modulation experiments have been performed with hydrated $\left(\mathrm{H}_{2} \mathrm{O}\right.$ and $\mathrm{D}_{2} \mathrm{O}$ $\sim 90 \%$ rel. humidity at $20^{\circ}$ ) multilayers of poly $(L$-lysine $)$ at $26^{\circ}$ and $35^{\circ}$ mean temperature, respectively. The modulation amplitudes were $\Delta T= \pm\left(1.0-1.5^{\circ}\right)$, with a period $\tau_{\mathrm{m}}=11 \mathrm{~min}$. The spectra shown in Fig. 15 demonstrate the considerable potential of modulation spectroscopy in resolving small differences and enabling access to complex kinetics. A detailed analysis will be given elsewhere [105]; however, some obvious features should be mentioned here (see also legend to Fig. 15). Fig. $15 \mathrm{~A}$ and $E$ present the stationary part of the spectra at $26^{\circ}$ hydrated by $\mathrm{H}_{2} \mathrm{O}$ and $\mathrm{D}_{2} \mathrm{O}$, respectively. Fig. $15 \mathrm{C}$ and $G$ show the corresponding spectra at $35^{\circ}$. Evidently, a conformational equilibrium (antiparallel pleated sheet with $\alpha$-helix and/or random) exists at $26^{\circ}$, whereas antiparallel pleated sheet conformation dominates at $35^{\circ}$. The former state is optimum for modulation experiments, since the temperature induced change of an equilibrium constant $K$,

$\frac{\mathrm{d} \ln K}{\mathrm{~d} T}=\frac{\Delta H}{R T^{2}}$

is most efficient with respect to changes in the equilibrium concentrations. $\Delta H$ denotes the standard enthalpy of reaction and $R$ the gas constant. Sign and magnitude of $\Delta H$ of each elementary step as well as the corresponding rate constants determine the degree of modulation $\rho_{i}$ and the phase angle $\phi_{i}$ (see Eqns. 31-34). As a consequence, phase-resolved spectra at a given modulation frequency $\omega_{m}=2 \pi / \tau_{m}$ and the dependence of the degree of modulation $\rho_{i}$ and phase angle $\phi_{i}$ on $\omega_{n}$ give detailed insight in the number and sequence of species affected by external stimulation [43][44][105]. Modulation spectra (Figs. 15B,D,F,H) are resolved at $0^{\circ}, 45^{\circ}, 90^{\circ}$, and $135^{\circ}$ phase shift with respect to the onset of $T$ stimulation. For better understanding, it should be noted that a positive band in the $0^{\circ}$-spectrum generally means formation, and a negative band disappearance of a species upon stimulation. Typical $\mathrm{H}_{2} \mathrm{O}$ and $\mathrm{D}_{2} \mathrm{O}$ bands $\left(\sim 3400, \sim 1640 \mathrm{~cm}^{-1}\left(\mathrm{H}_{2} \mathrm{O}\right), \sim 2500, \sim\right.$ $1200 \mathrm{~cm}^{-1}\left(\mathrm{D}_{2} \mathrm{O}\right)$ ) have positive signs. Consequently, water binding from the atmosphere $\left(\mathrm{N}_{2} / \mathrm{H}_{2} \mathrm{O}, \mathrm{D}_{2} \mathrm{O}, \sim 90 \%\right.$ rel. humidity at $20^{\circ}$ ) is enhanced by $T$-stimulation. This is reasonable, since the stimulation cycle started at minimum temperature $T-\Delta T$ followed by an increase to the maximum temperature $T+\Delta T$. Pleafed sheet bands have opposite sign, indicating that the conformation is favoured at elevated temperature. Furthermore, it should be mentioned that a modulation band reaching maximum amplitude at phase angle $\phi$ $=\phi_{i}$, is erased at $\phi=\phi_{i} \pm 90^{\circ}$. Therefore, if in a composed band e.g. amide $\mathrm{I}, \mathrm{I}(\sim 1650$ $\left.\mathrm{cm}^{-1}\right)$, amide II' $\left(\sim 1540 \mathrm{~cm}^{-1}\right)$, amide II ( 
$\sim 1450 \mathrm{~cm}^{-1}$ ) the components respond with different kinetics to the external stimulation, the shape of the overall band should depend on the reference phase angle (here: $0^{\circ}, 45^{\circ}, 90^{\circ}, 135^{\circ}$ ). This is indeed the case in the spectra presented in Fig. 15, documenting that phase resolved spectra may give a detailed und unambiguous insight into complex bands. Working with a phase resolution of $9^{\circ}$ enables data presentation in a 3D plot (Fig. 16). The experimental data are the same as used in Fig. $15 F$. It should be noted that all absorption bands of a given species must exhibit the same phase angle, i.e. if a maximum of a modulation band appears at a different phase angle, it unambiguously results from a different species.

\section{Prospects}

This review article has featured general and special aspects of IR ATR spectroscopy. Results justify the hopes that this technique may become a serious complement in drug design and drug delivery. Of course, there is need for further refinements of experimental methods and theory. In this context, it is planed to introduce more general ultrastructural models adequate for dichroic analysis of oriented poly- and oligopeptides, as well as of hydrocarbon chains. For that purpose, it is essential to get informations on energetically reasonable local conformations, i.e. on local distibution functions. Such data are available from molecular modeling. On the other hand, data on molecular structure derived from IR ATR can be used to filter the variety of possible structures derived from molecular modeling. Such concerted investigations are not limitted to immobilized oriented samples. Molecular structures can be studied in solution, too, provided the concentration of the solute is greater than $1-10 \mathrm{~mm}$, depending on the molar absorption coefficients. Especially external parameter modulation techniques will be applied in a variety of different forms enabling the detection and analysis of conformational equilibria in immobilized and dissolved samples.

The author thanks Jeannette Goette and Martin Müller for making results available prior to publication, and to Marianna Fringeli and Thomas Meyer for technical assistence. Financial support by the Swiss National Science Founda tion, (project No 31-27772.89) is kindly acknowledged. Acknowledgement for technical support, see [99].

\section{Received: September 19, 1991}

[1] P.R. Griffiths, 'Chemical Infrared Fourier Transform Spectroscopy', John Wiley and Sons, Inc., New York, 1975.

[2] U.P. Fringeli, H.-J. Apell, M. Fringeli, P. Läuger, Biochim. Biophys. Acta 1989, 984 , 301.

[3] K. Brandenburg, U. Seydel, Eur. J. Biochem. 1988, 16, 83.

[4] M.S. Braimann, K.J. Rothschild, Eur. J. Biochem. 1988, 17, 541 .

[5] U.M. Ganter, W. Gärtner, F. Siebert, Eur. Biophys. J. 1990, 16, 295.

[6] A. Barth, W. Kreutz, W. Mäntele, Biochim. Biophys. Acta 1991, 1057, 115.

[7] A. Blume, W. Hübner, G. Messner, Biochemistry 1988, 27, 8239.

[8] T.I.Lotta, I.S. Salonen, J.A. Virtanen, K.K. Ecklund, P.K.J. Kinnunen, Biochemistry 1988, 27, 8158 .

[9] F. Tanfani, A. Ambrosini, G. Albertini, E. Bertolini, G. Curatola, G. Zolese, Chem. Phys. Lipids 1990, 55, 179.

[10] H.J. Casal, R.L. McElhaney, Biochemistry $1990,29,5423$

[11] W.K. Surewicz, H.H. Mantsch, Biochim. Biophys. Acta 1988, 952, 115.

[12] H.-U. Gremlich, U.P. Fringeli, R. Schwyzer, Biochemistry 1984, 23, 1808 .

[13] P.L. Poole, D.J. Barlow, Biopolymers 1986, 25,317 .

[14] P.I. Haris, D. Chapman, R.A. Harrison, K.F.Smith, S.J.Perkins, Biochemistry 1990, 29, 1377.

[15] F. Kopp, U.P. Fringeli, K. Mühlethaler, Hs.H. Günthard, Biophys. Struct. Mech $1975,1,75$.

[16] R.M. Gendreau, Trends Anal.Chem. 1986 , 5,68 .

[17] R.J. Jakobsen, F.M. Wasacz, K.B. Smith 'Chemical, Biological and Industrial Applications of Infrared Spectroscopy', Ed J.R. Durig, John Wiley \& Sons, New York, 1985, p. 199.

[18] E. Goormaghtigh, R. Brasseur, P. Huarz, J.M. Ruysschaert, Biochemistry 1987, 26, 1789.

[19] A. Mellier, 'Spectroscopy of Biological Molecules, New. Advances' Eds. Schmid. Schneider, and Siebert, J. Wiley, Chichester, 1988, p. 173.

[20] J.L.R. Arrondo, A. Muga, J. Castresana, C. Bernabeu, F.M. Goni, FEBS Lett. 1989, $252,118$.

[21] J. Villalain, A. Ortiz, J.C. Gomez-Fernandez, Biochim. Biophys. Acta 1988, 941, 55.

[22] D.G. Cornell, R.A. Dluhy, M.S. Briggs, C.J.McKnight, L.M. Gierasch, Biochemis try 1989, 26, 2789.

[23] A.B. Chetverin, E.V. Brazhnikov, J. Biol. Chem. 1985, 260, 7817

[24] D. Naumann, C. Schu ltz, J. Born, F. Rogge, H. Labischinski, Microchim. Acta 1988, I, 379.

[25] B. Kleffel, R.M. Garavito, W. Baumeister, J.P. Rosenbusch, EMBO J. 1985, 4, 1589

[26] R.A. Dluhy, N.A. Wright, P.R. Griffiths, Appl. Spectrosc. 1988, 42, 138.

[27] S. Frei, L. Tamm, Biophys. J. 1991, 60 , 922.

[28] R. Buchet, S. Varga, N.W. Seidler, E. Molnar, A. Martonosi, Biochim. Biophys. Acta 1991, 1068, 201.
29] A. Dong, P. Huang, W.S. Caughey, Biochemistry 1990, 29, 3303.

[30] H. Vogel, F. Jahnig, V. Hoffmann, J. Stümpel, Biochim. Biophys. Acta 1983, 733, 201.

[31] A.T. Tu, 'Spectroscopy of Biological Systems', Eds. R.J.H. Clark and R.E. Hester, John Wiley \& Sons Lid., New York, 1986, p. 47.

[32] F.S. Parker, 'Applications of Infrared, Raman and Resonance Raman Spectroscopy in Biochemistry', Plenum, New York, 1983.

[33] J. Bandekar, D.J. Evans, S. Krimm, S.J. Leach, M.M. Aine, G. Minamasian, G. Nemethy, M.S. Pottle, H.A. Scheraga, E.R. Stinson, R.W. Woody, Int.J. Peptide Prot. Res. 1982, 19, 187; S. Krimm, J. Bandekar, Adv. Protein Chem. 1986, 38, 181.

[34] M. Pezolet, L. Duschesneau, P. Bougis, J. Faucon, J. Dufourcg, Biochim. Biophys. Acta 1982, 704, 515.

[35] J.W. Brauner, R. Mendelsohn, F.G. Prendergast, Biochemistry 1987, 26, 8151.

[36] W.-J. Yang, P.R. Griffiths, D.M. Byler, H. Susi, Appl. Spectrosc. 1985, 39, 282.

[37] F. Kopp, P.A. Cuendet, in 'Liquid Crystals of One- and Two-Dimensional Order', Eds. W. Helfrich and G. Heppke, Springer, Berlin, 1980, p. 361.

[38] W. Hübner, H.H.Mantsch, Biophys.J. 1991, $59,1261$.

[39] M.Dunãch, E. Pedròs, E. Seigneuret, J.-L. Rigaud, J. Biol. Chem. 1988, 263, 7555.

[40] E. Okamura, J. Umemura, T. Takenaka, Biochim. Biophys. Acta 1990, J025, 94.

[41] For a review see: Chemical, Biochemical and Environmental Sensors, R.A. Lieberman, M.T. Wlodarczyk, SPIE 1989, $1 / 72$.

[42] U.P. Fringeli, Hs.H. Günthard, Appl. Opt. 1971, 10,819 .

[43] M. Forster, U.P. Fringeli, Hs.H. Günthard, Helv. Chim. Acta 1973, 389.

[44] Hs.H. Günthard, Ber. Bunsenges. Phys. Chem. 1974, 76, 1110.

[45] U.P.Fringeli, Hs.H. Günthard, 'Membrane Spectroscopy', Ed. E. Grell, 'Molecular Biology, Biochemistry and Biophysics', Springer, Berlin, 1981, Vol. 31, p. 270.

[46] U.P. Fringeli, 'In Situ Infrared Attenuated Total Reflection Membrane Spectroscopy', in 'Internal Reflection Spectroscopy: Theory and Application', Ed. F.M. Mirabella, Marcel Dekker, New York, 1992. in press.

[47] N.J. Harrick, 'Internal Reflection Spectroscopy', Harrick Sci. Corp., Ossining, New York, 1979.

[48] F. Goos, H. Lindberg-Hänchen, Ann. Phys. $1949,5,251$.

[49] M. Born, E. Wolf, 'Principles of Optics', Pergamon Press, Oxford, 1983.

[50] R.D.B. Fraser, T.P. MacRae, 'Conformations in Fibrous Proteins and Synthetic Polypeptides', Academic Press, New York, 1973, Chapt. 5.

[51] R.Zbinden, 'Infrared Spectroscopy of High Polymers', Academic Press, New York, 1964.

[52] J. Michl, E.W. Thulstrup, 'Spectroscopy with Polarized Light', VCH Publ. Inc., New York, 1986.

[53] E.B. Wilson, J.C. Decius, P.C. Cross, 'Molecular Vibrations. The Theory of IR and Raman Vibrational Spectra', Mc GrawHill, New York, 1955. 
[54] O. Kratky, Kolloid Z. 1933, 64, 213.

[55] U.P. Fringeli, M. Schadt, P. Rihak, Hs.H. Günthard, Z. Naturforsch., A 1976, 31, 1098.

[56] A. Saupe, Z.Naturforsch., A 1964, 19, 161.

[57] J. Seelig, Q. Rev. Biophys. 1977, 10, 353

[58] E. Egberts, H.J.C. Berendsen, J. Chem. Phys. 1988, 89, 3718.

[59] H.-U. Gremlich, U.P. Fringeli, R. Schwyzer, Biochemistry 1983, 22, 4257.

[60] U.P. Fringeli, P. Leutert, H. Thurnhofer, M. Fringeli, M.M. Burger, Proc.Natl.Acad. Sci. U.S.A. 1986, 83, 1315.

[61] J. Goette, 'ATR-IR-spektroskopische Untersuchung der Lokalanästhetika-Phospholipid-Modellmembran-Interaktion und dampfdruckosmometrische Bestimmung der Assoziation von Lokalanästhetika in wässriger Lösung', ETH-Dissertation, Nr. 9721, 1992, and J. Goette, X. Perlia, U.P. Fringeli, in preparation.

[62] U.P. Fringeli, 'Biologically Active Molecules', Ed. U.P. Schlunegger, Springer, Berlin, 1989, p.241.

[63] U.P. Fringeli, Nachr. Chem. Tech. Lab. $1985,33,708$

[64] U.P. Fringeli, J. Membrane Biol. 1980, 54, 203.

[65] U.P. Fringeli, Hs.H. Gunthard, Biochim Biophys. Acta 1976, 450, 101

[66] U.P. Fringeli, P. Ahlström, C. Vincenz, M.Fringeli, 'Fourier and Computerized IR Spectroscopy', Eds. J. Grasselli and D. Cameron, SPIE 1985, 553, 234.

[67] P. Hofer, Kinetische und strukturelle Untersuchungen an der Acety lcholinesterase (EC 3.1.1.7) PhD Thesis, ETH 7112, 1982.

[68] U.P. Fringeli, P. Hofer, Neurochem. Int. 1980, 2, 185.

[69] K.B. Blodgett, I. Langmuir, Phys. Rev. $1937,51,964$

[70] H. Kuhn, D. Möbius, H. Bucher, 'Techniques of Chemistry', Eds. A. Weissberger and B. Rossiter, John Wiley \& Sons, New York, 1972, I, Part IIIb, p. 577.

[71] E. Haidl, Diploma Work, University of Vienna, 1988

[72] K. Fendler, E. Grell, M. Haubs, E. Bamberg, EMBO J. 1985, 4, 3079.

[73] R. Borl inghaus, H.-J. Ape 1 1, P. Läuger, $J$. Membrane Biol. 1987, 97, 161.

[74] P. Hofer, U.P. Fringeli, Biophys. Struct. Mech. 1979, 6, 67.

[75] M. Schöpflin, U.P. Fringeli, X. Perlia, $J$. Am. Chem. Soc. 1987, 189, 2375

[76] Y. Boulanger, S. Schreier, L.C. Leitch, I.C.P. Smith, Can. J. Biochem. 1980, 58, 986.

[77] A. Seelig, P.R. Allegrini, J. Seelig, Biochim. Biophys. Acta 1988, 939, 267.

[78] Y. Boulanger, S. Schreier, I.C.P. Smith, Biochemistry 1981, 20, 6824

[79] M. Auger, H.C. Jarrell, I.C.P. Smith, Biochemistry 1988, 27, 4660 .

[80] For a comprehensive discussion of the literature on $\mathrm{LA} / \mathrm{membrane}$ interaction see [61].

[81] D.G. Cameron, H.L. Casal, H.H. Mantsch, Biochemistry 1980, 19, 3665.

[82] For charged drug molecules, the concentration at the membrane-electrolyte interface $c_{i}$ may differ from the bulk concentration $c b$. The intrinsic partition coefficient is, therefore, defined as $P_{\text {int }}=\lim _{c_{b} \rightarrow 0} \frac{c_{m}}{c_{i}}$.
[83] H.-D. Bäuerle, J.Seelig,Biochemistry 1991, $30,7203$.

[84] H.P. Limbacher, G.D. Blickenstaff, J.H. Bowen,H.H.Wang, Biochim. Biophys.Acta 1985, 812, 268.

[85] P. Schlieper, L. Michaelis, Biophys. Struct. Mech. 1983, 10, 1.

[86] D. Papahadjopoulos, K. Jacobson, G.Poste, G. Shepherd, Biochim. Biophys. Acta 1975, 394, 504.

[87] A. Cao, E. Hantz-Brachet, B Azize, E. Taillandier, G. Perret, Chem. Phys. Lipids 1991, 56, 225.

[88] A.G. Lee, Biochim. Biophys. Acta 1978, $514,95$.

[89] Y. Kaminoh, H. Kamaya, I . Ueda, Biochim. Biophys. Acta 1989, 987, 63.

[90] S. Ohki, Biochim. Biophys. Acta 1984,777, 56.

[91] S. McLaughlin, H. Harary, Biochemistry 1976, $15,1941$.

[92] S. McLaughlin, 'Molecular Mechanisms of Anesthesia', Ed. B.R. Fink, 'Progress in Anesthesiology', Raven Press, New York, 1975, Vol. 1, p. 193.

[93] E.K. Rooney, A.G. Lee, Biochim. Biophys. Acta 1983, 732, 428

[94] M.R. Eftink, R.K. Puri, M.D. Ghahramani, Biochim. Biophys. Acta 1985, 813, 137.

[95] B.W. Ninham, Y.A. Parsegian, J. Theor. Biol. 1971, 31, 405 .

[96] G. Cevc, D. Marsh, 'Phospholipid Bilayers', John Wiley \& Sons, New York, 1987, p. 126. This book gives a comprehensive review of physico-chemical methods.

[97] B.O. Seraphin, Proceedings on the $1 \mathrm{st}$ Conf. on Modulation Spectroscopy, North Holland Publ., Amsterdam, 1973.

[98] L.A. Nafie, D.W. Vidrine, 'Double modulation Fourier transform spectroscopy', in 'Fourier Transform IR spectroscopy', Academic Press, New York, 1982, Vol. 3, p. 83.

[99] A Bruker IFS 48 FTIR Spectrometer has been modified for low frequency modulation spectroscopy. The project was performed in collaboration with Bruker Analytische Messtechnik, Karlsruhe (J. Gast, A. Simon, J. Gronholz), Spectrospin $A G$, Fällanden (N. Spirig, H.W. Tschopp). It was financially supported by the Kommission zur Förderung der wissenschaftlichen Forschung $(K W F)$, Bern (Projects No. 1895.1, 2245.1)

[100] H. Susi, S.N. Timasheff, L. Stevens, $J$. Biol. Chem. 1967, 242, 5460.

[101] T.-J. Yu, J.L. Lippert, W.L. Peticolas, Biopolymers 1973, 12, 2161.

[102] N. Greenfield, G.D. Fasman, Biochemistry $1969,8,4108$

[103] U. Shmueli, W. Traub, J. Mol. Miol. 1965, 12, 205.

[104] S.D. Swanson, R.G. Bryant, Biopolymers $1991,31,967$

[105] M. Müller, Möglichkeiten und Grenzen der Infrarot-Spektroskopie bei der Sekundärstrukturaufklärung von Proteinen und Peptiden, ETH-Dissertation 1992, and U.P. Fringeli, M. Müller, in preparation. 